\title{
Phase-Based Impedance Control of a Powered Knee-Ankle Prosthesis for Tuning-Free Locomotion over Speeds and Inclines
}

\author{
T. Kevin Best, Cara G. Welker, Elliott J. Rouse, and Robert D. Gregg
}

\begin{abstract}
Most impedance-based walking controllers use a finite state machine (FSM) with dozens of user-specific parameters that need to be manually tuned by technical experts. These parameters are only optimal near the task (e.g., walking speed and incline) at which they were tuned, resulting in decreased performance as task inevitably varies. This paper presents a tuningfree, phase-based controller that uses a hybrid combination of continuously-variable impedance control during stance and kinematic control during swing to enable biomimetic locomotion over a continuum of tasks. After generating a data-driven model of variable joint impedance with convex optimization, we implement a novel task-invariant phase variable and real-time estimates of speed and incline to enable the controller to autonomously adapt to task variation. Experiments with an amputee participant using a powered knee-ankle prosthesis show that our tuningfree controller 1) features highly-linear phase estimates and accurate task estimates, 2) produces more biomimetic joint work trends compared to a hand-tuned FSM impedance controller, and 3) achieves lower kinematic and kinetic error than the FSM impedance controller in 7 of 8 tested metrics. Our data-driven control approach may allow easier clinical implementation of variable-activity powered knee-ankle prostheses by replicating biological behavior across tasks without expert tuning.
\end{abstract}

Index Terms-Prostheses, Impedance Control, Optimization

\section{INTRODUCTION}

To perform activities that require net-positive energy, such as ascending ramps and stairs, passive prosthesis users must supply supplemental power from intact joints [1], leading to secondary complications including increased energy expenditure [2], osteoarthritis [3], and lower back pain [4]. While powered prostheses can help avoid these complications by performing net-positive work [1], [5]-[8], designing prosthetic control systems for diverse environments remains a challenge.

Impedance control is a common strategy in lower-limb wearable robotics because of its simplicity and ability to produce behaviors that are similar to human biology, such as compliantly controlled interaction with the ground [9] and short-range dynamics similar to muscles [10]. Further, empirical studies have shown that ankle joint dynamics during walking are well described with an impedance controller [11][13]. A standard impedance controller calculates joint torque $\tau$ based on a joint angle $\theta$ and joint velocity $\dot{\theta}$ as

$$
\tau=-K\left(\theta-\theta_{\text {eq }}\right)-B \dot{\theta},
$$

This work was supported by the National Institute of Child Health \& Human Development of the NIH under Award Number R01HD094772. This work was also supported by the National Science Foundation under Award Numbers 1846969 and 1949346 . The content is solely the responsibility of the authors and does not necessarily represent the official views of the NIH or NSF.

T. Kevin Best, Cara G. Welker, and Robert D. Gregg are with the Department of Electrical Engineering and Computer Science and the Robotics Institute, University of Michigan, Ann Arbor, MI 48109. Elliott J. Rouse is with the Department of Mechanical Engineering and the Robotics Institute, University of Michigan, Ann Arbor, MI 48109. Contact: \{tkbest, cgwelker, ejrouse, rdgregg\}@umich. edu where $K, B$, and $\theta_{\text {eq }}$ are parameters defining the joint's stiffness, damping, and equilibrium angle, respectively.

Traditional methods of impedance control for lower-limb prostheses involve segmenting the gait cycle into discrete subphases, where each sub-phase has its own constant values of $K, B$, and $\theta_{\text {eq }}$. Researchers manually tune the impedance parameters in each sub-phase until the observed gait is satisfactory [5], [11], [14]-[17]. Switching between sub-phases is controlled by a finite state machine (FSM) with transition criteria based on sensor readings (e.g., elapsed time, leg loading, joint angles, etc.). Like the impedance parameters, these transition criteria are often experimentally tuned for an individual's gait by a technical expert. More elaborate impedance value representations have been suggested [16][19], but these methods still required manual, expert tuning.

Joint kinematics and kinetics vary based on the ground incline and walking speed [20], [21] (together termed the user's task). Therefore, the necessary impedance parameters and state machine transition criteria also vary. For a standard FSM impedance controller to operate over a wide array of tasks, many tunable parameters are required. For example, one state-of-the-art impedance controller for five ambulation modes required a total of 140 tunable parameters [16]. While only a portion of these parameters were considered necessary to tune, the device's configuration and tuning still required the researchers up to five hours to complete. To avoid the expert tuning burden, other work aimed to eliminate manual tuning by using biological quasi-stiffness curves [17], [22]-[24] or using machine learning to perform online parameter tuning [25], [26]. However, many of these approaches were limited to level-ground walking. Further, while [24]-[26] addressed the problem of tuning the impedance parameters, their approaches still required tuning the FSM switching criteria.

In contrast to the standard FSM-based impedance control paradigm, some authors have suggested using continuous functions to define the impedance parameters and how they evolve over the gait cycle [27]-[30]. In general, controllers that continually vary a robot's output mechanical impedance with time are known as variable impedance controllers [31]. Biomechanical principles suggest that human joints behave like variable impedance controllers [32] and empirical studies have observed this behavior at the ankle joint during walking [11]-[13]. Therefore, variable impedance control may offer a biomimetic solution for controlling powered prosthetic legs. However, how to appropriately define the variable impedance functions to realize walking gaits remains an open question.

A variable impedance controller was suggested in [27] using linear functions for stiffness and damping during stance. The linear functions were hand-tuned and held constant regardless of task. The variable impedance control method in [28] 
eliminated tuning altogether by using able-bodied kinematic data to generate continuous impedance parameter functions of phase. However, this method was limited to the knee joint, did not consider joint kinetics, and was never experimentally validated. Recently, [29] proposed a similar variable impedance controller where ankle stiffness and damping were defined as polynomials in phase, and the coefficients defining the polynomials were identified using constrained least squares with an able-bodied kinematic and kinetic dataset. The authors utilized piecewise-constant equilibrium angles and demonstrated continuous stiffness and damping expressions that produced satisfactory gait with a post-optimization tuning protocol. This work was later extended to include variable inclines and a phase variable parameterization of stiffness and damping based on the phase portrait of the thigh angle and its integral [30]. However, this phase variable is known to have challenges with non-steady walking [33], and changes in impedance associated with walking speed were not considered. The authors of [30] also note that their method of identifying the impedance parameters is non-convex, which does not guarantee a globally optimal solution [34] for their controller.

This paper addresses these limitations by presenting a new phase-based, task-adaptive walking controller built on a hybrid combination of continuously-variable impedance control during stance and kinematic control during swing (Fig. 1). Stance impedance control allows the controller to regulate the dynamic interaction between the user and the ground while swing kinematic control provides the user with indirect volitional control over foot position. We use an able-bodied kinematic and kinetic dataset [21] with constrained convex optimization to create a continuous model of stance joint stiffness, damping, and equilibrium angle, each parameterized by phase, walking speed, and ground incline. Paired with an analogous model of swing joint kinematics, our hybrid controller produces a biomimetic gait across varying tasks based on real-time phase and task estimates.

While similar to [30], our approach is distinct in multiple important ways. First, the convex optimization formulation provides an approximation of the globally optimal impedance parameter functions. Second, our variable impedance model includes a continuous function for equilibrium angle, mirroring the continuous progression of biological joint dynamics [11][13]. Third, this variable impedance model is further parameterized by walking speed, which is critical to reproducing normative gait energetics [35]. Fourth, we estimate the task variables in real-time, making the system fully autonomous. Fifth, we use a phase variable that is more robust to variable speed and incline behavior than prior phase variable approaches [30], [33], [35], [36]. And sixth, we demonstrate that our approach works for a novel above-knee amputee participant without manual tuning over a range of tasks, resulting in lower kinetic and kinematic error compared to a standard hand-tuned impedance controller in most metrics.

In summary, this work provides the following novel contributions: 1) A convex, data-driven framework to calculate joint stiffness, damping, and equilibrium angle during the stance phase of gait as continuous functions of phase, walking speed, and incline. 2) An improved phase variable that avoids

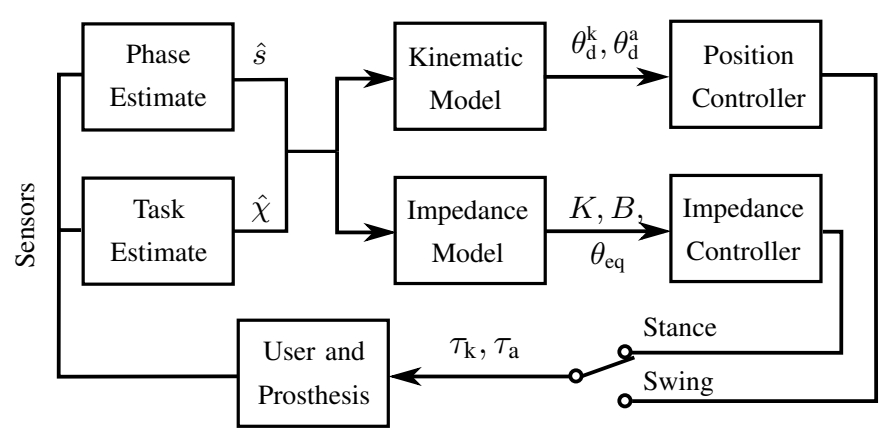

Fig. 1. A block diagram of the Hybrid Kinematic Impedance Controller proposed in this work. Real-time estimates of gait phase $\hat{s}$ and task $\hat{\chi}$ define desired joint impedance parameters $K, B, \theta_{\mathrm{eq}}$ and joint angles $\theta_{\mathrm{d}}^{\mathrm{k}}, \theta_{\mathrm{d}}^{\mathrm{a}}$ using data-driven models. Depending on if the user is in stance or swing, the torque commands $\tau_{\mathrm{k}}, \tau_{\mathrm{a}}$ are calculated using either an impedance controller or a position controller, respectively.

kinematic singularities and is robust to a diverse family of thigh trajectories at variable walking speeds and inclines. 3) An experimental validation with an above-knee amputee participant demonstrating that the hybrid variable impedance controller produces more biomimetic joint work trends as incline varies compared to a hand-tuned FSM impedance controller, and lower kinematic and kinetic error than the FSM impedance controller in 7 of 8 tested metrics.

The remainder of this paper is organized as follows. Section III details the optimization process for calculating the continuous impedance parameter models for stance. Then in Section III]. we define the hybrid controller based on this impedance model for stance and our previous kinematic model [20] for swing. We further define a new task-invariant phase variable and incline and speed estimation methods that allow the controller to operate fully autonomously. Section IV] describes the experiments in which a participant with an above-knee amputation compared the controller proposed in this work with a hand-tuned FSM impedance controller. Finally, in Section V we discuss the results, study limitations, and future work.

\section{VARiable Impedance Model for Stance}

\section{A. Model Framework}

To use impedance control for the stance phase of the gait cycle in a continuous, phase-based control framework, we require a model analogous to the kinematic model developed in [20] that describes how the impedance parameters $(K, B$, and $\theta_{\text {eq }}$ ) evolve during stance. Specifically, we require the impedance parameter model to be continuously parameterized by both gait phase $s$ and task $\chi=(\nu, \gamma)$, where task is defined by the current walking speed $\nu$ and ground incline $\gamma$ over the range $-10 \leq \gamma \leq 10$ deg and $0.8 \leq \nu \leq 1.2 \mathrm{~m} / \mathrm{s}$.

A model that meets these criteria can be constructed from linear combinations of phase-varying polynomials, where the linear combination weights depend on task. Polynomial functions of phase are useful to model parameter progression during stance because they are simply parameterized and can represent arbitrary aperiodic signals. We use fourth order polynomials $(d=4)$, as they allow sufficient flexibility to model the parameter behavior without overfitting. Once the appropriate polynomial functions are identified for individual 
tasks in a dataset, bilinear interpolation can be used to create a unified, continuous model with task and phase inputs

First, we define task-specific polynomial functions that represent how the parameters vary during stance for a set of fixed tasks. For convenience, let $s_{\mathrm{st}}$ be the stance phase (i.e., $s_{\mathrm{st}}=s / s_{\mathrm{TO}}$, where $s_{\mathrm{TO}}$ is the phase at toe-off). Then, the impedance parameters for the $p$-th fixed task $\chi_{p}$ are

$$
K_{\chi_{p}}=\sum_{i=0}^{d} k_{i p} s_{\mathrm{st}}^{i}, B_{\chi_{p}}=\sum_{i=0}^{d} b_{i p} s_{\mathrm{st}}^{i}, \theta_{\mathrm{eq}, \chi_{p}}=\sum_{i=0}^{d} e_{i p} s_{\mathrm{st}}^{i},
$$

where $\kappa_{\chi_{p}}=\left\{\left(k_{i p}, b_{i p}, e_{i p}\right) \mid i \in\{0, \ldots, d\}\right\}$ is a set of constant coefficients. Then, the coefficients $\kappa_{\nu \gamma}$ defining the impedance parameter trajectories for an arbitrary task $(\nu, \gamma)$ are calculated through bilinear interpolation of its four nearest neighboring tasks $\kappa_{\nu_{n}, \gamma_{n}}$, where $\nu_{n} \in\left\{\nu_{1}, \nu_{2}\right\}, \gamma_{n} \in\left\{\gamma_{1}, \gamma_{2}\right\}$. For all $j$ elements in $\kappa_{\nu \gamma}$, this interpolation is

$$
\kappa_{\nu \gamma}^{j}=\frac{\left[\begin{array}{ll}
\nu_{2}-\nu & \nu-\nu_{1}
\end{array}\right]}{\left(\nu_{2}-\nu_{1}\right)\left(\gamma_{2}-\gamma_{1}\right)}\left[\begin{array}{ll}
\kappa_{\nu_{1} \gamma_{1}}^{j} & \kappa_{\nu_{1} \gamma_{2}}^{j} \\
\kappa_{\nu_{2} \gamma_{1}}^{j} & \kappa_{\nu_{2} \gamma_{2}}^{j}
\end{array}\right]\left[\begin{array}{l}
\gamma_{2}-\gamma \\
\gamma-\gamma_{1}
\end{array}\right] .
$$

Finally, using $\kappa_{\nu \gamma}$ and (2) evaluated at the current stance phase $s_{\text {st }}$, the impedance parameters are calculated. Therefore, the model is fully defined once each task-specific set of coefficients $\kappa_{\chi_{p}}$ is calculated.

\section{B. Model Fitting}

We use an optimization-based approach to fit the model for each task $\chi_{p}$ to a dataset of able-bodied walking [21]. The dataset contains kinematic and kinetic joint information from 10 participants walking at steady-state at 15 distinct points in the task space $(\gamma \in\{-10,-5,0,5,10\} \mathrm{deg}, \nu \in$ $\{0.8,1.0,1.2\} \mathrm{m} / \mathrm{s})$. Therefore, for each task $\chi_{p}$, we constructed an optimization problem to identify the set of impedance parameter coefficients $\kappa_{\chi_{p}}^{*}$ that, when used in (2) and the impedance control equation, best reproduced the massnormalized joint torques $\tau$ in the dataset given the dataset kinematics $(\theta, \dot{\theta})$ over all $n$ data points at $\chi_{p}$ :

$$
\begin{array}{r}
\kappa_{\chi_{p}}^{*}=\arg \min \frac{1}{n}\|\tau-\hat{\tau}\|_{2}^{2}, \\
\text { where } \hat{\tau}=K_{\chi_{p}}\left(\theta_{\text {eq }, \chi_{p}}-\theta\right)-B_{\chi_{p}} \dot{\theta} .
\end{array}
$$

1) Solution Approximation: As written, (4) is difficult to solve, as the product $K_{\chi_{p}} \theta_{\mathrm{eq}, \chi_{p}}$ is nonlinear in the unknown parameters, and the overall objective function is non-convex. To avoid this problem, we solve a similar, convex problem and use its solution to approximate a solution to (4). First, we combine the product of $K_{\chi_{p}}$ and $\theta_{\mathrm{eq}, \chi_{p}}$ into a new, higherorder polynomial $\delta_{\chi_{p}}$ with independent coefficients $\delta_{i p}$ :

$$
K_{\chi_{p}} \theta_{\mathrm{eq}, \chi_{p}}=\sum_{i=0}^{d} k_{i p} s_{\mathrm{st}}^{i} \sum_{i=0}^{d} e_{i p} s_{\mathrm{st}}^{i}=\sum_{i=0}^{2 d} \delta_{i p} s_{\mathrm{st}}^{i}=\delta_{\chi_{p}} .
$$

By treating the $\delta_{i p}$ terms as independent from the $k_{i p}$ terms, the impedance equation for $\hat{\tau}$ becomes linear in the unknown parameters $k_{i p}, b_{i p}$, and $\delta_{i p}$. We can then write the modified optimization problem as a standard quadratic program, defining a new argument vector $x \in \mathbb{R}^{4 d+3 \times 1}$ as

$$
x=\left[k_{0 p}, \ldots, k_{d p}, b_{0 p}, \ldots, b_{d p}, \delta_{0 p}, \ldots, \delta_{2 d p}\right]^{\top} .
$$

Let $\alpha_{j} \in \mathbb{R}^{4 d+3 \times 1}$ be defined for each data point $j$ as

$$
\alpha_{j}=\left[-\theta_{j} s_{j}^{0}, \ldots,-\theta_{j} s_{j}^{d},-\dot{\theta}_{j} s_{j}^{0}, \ldots,-\dot{\theta}_{j} s_{j}^{d}, s_{j}^{0} \ldots, s_{j}^{2 d}\right]^{\top} .
$$

Then, the objective function $L\left(\kappa_{\chi_{p}}\right)$ from 4 becomes

$$
L\left(\kappa_{\chi_{p}}\right)=\frac{1}{n}\|\tau-\hat{\tau}\|_{2}^{2}=\frac{1}{n} \sum_{j=1}^{n} \tau_{j}^{2}-f^{\top} x+\frac{1}{2} x^{\top} H x,
$$

where

$$
H=\frac{2}{n} \sum_{j=1}^{n} \alpha_{j} \alpha_{j}^{\top}, \quad f=\frac{2}{n} \sum_{j=1}^{n} \tau_{j} \alpha_{j} .
$$

2) Constraints and Regularization: To prevent elements of $x$ from dominating the solution, we added a diagonal regularization matrix $R=\operatorname{diag}(\lambda) \in \mathbb{R}^{4 d+3 \times 4 d+3}$ to $H$ to penalize the $L_{2}$ norm of $x$. The $k$-th diagonal entry for the regularization weight was $\lambda_{k}=1 e^{-5}$ for terms associated with stiffness and damping and $\lambda_{k}=1 e^{-2}$ for the $\delta_{i}$ terms.

Next, we added a constraint matrix $A$ to ensure that $K_{\chi_{p}}$ and $B_{\chi_{p}}$ remained within ranges that were both physiological realistic and feasible for the prosthesis to render. Namely, the stiffness function was constrained above $1.5 \mathrm{Nm} / \mathrm{rad} / \mathrm{kg}$ and the damping function between 0.01 and $1.0 \mathrm{Nms} / \mathrm{rad} / \mathrm{kg}$. In addition, prosthesis users noted during preliminary experiments that a low stiffness at heelstrike was unsettling, as they were accustomed to a locked knee during early stance with their take-home prostheses. Therefore, a minimum heelstrike stiffness of $3.0 \mathrm{Nm} / \mathrm{rad} / \mathrm{kg}$ was added to increase participants' confidence in the device. To enforce these constraints, we discretized stance phase into $n_{j}$ points in the range $[0,1]$. We constructed a constraint matrix $A \in \mathbb{R}^{3 n_{j} \times 4 d+3}$ from submatrices $A_{s} \in \mathbb{R}^{n_{j} \times d+1}$, defined as

$$
A_{s}=\left[\begin{array}{ccc}
s_{1}^{0} & \ldots & s_{1}^{d} \\
\vdots & \ddots & \vdots \\
s_{n_{j}}^{0} & \ldots & s_{n_{j}}^{d}
\end{array}\right], \quad A=\left[\begin{array}{ccc}
-A_{s} & \mathbf{0} & \mathbf{0} \\
\mathbf{0} & -A_{s} & \mathbf{0} \\
\mathbf{0} & A_{s} & \mathbf{0}
\end{array}\right] \text {. }
$$

A column vector $b \in \mathbb{R}^{3 n_{j} \times 1}$ contained $n_{j}$ copies of the minimum stiffness and damping and maximum damping values, with the first term modified for the heelstrike constraint:

$$
b=[3.0,1.5, \ldots, 1.5,0.01, \ldots, 0.01,1.0, \ldots, 1.0]^{\top} .
$$

Finally, we arrived at the full quadratic program (QP), with the positive (sum-of-squares torque) offset in (8) neglected without loss of generality:

$$
\begin{array}{ll}
\underset{x}{\operatorname{minimize}} & \frac{1}{2} x^{\top}(H+R) x-f^{\top} x \\
\text { subject to } & A x \leq b .
\end{array}
$$

We solved the QP in (12) for each subject and task $\chi_{p}$ combination in the dataset $(N=150)$ using the MATLAB Optimization Toolbox. Then, we approximated the solution to the original problem (4) by fitting a $d$ order polynomial to $\delta_{\chi_{p}}\left(s_{\mathrm{st}}\right) / K_{\chi_{p}}\left(s_{\mathrm{st}}\right)=\theta_{\mathrm{eq}, \chi_{p}}\left(s_{\mathrm{st}}\right)$. We assumed the polynomial order was sufficiently high to approximate the rational function $\delta_{\chi_{p}}\left(s_{\mathrm{st}}\right) / K_{\chi_{p}}\left(s_{\mathrm{st}}\right)$ without significant information loss. This assumption was validated by the model's low reconstruction 

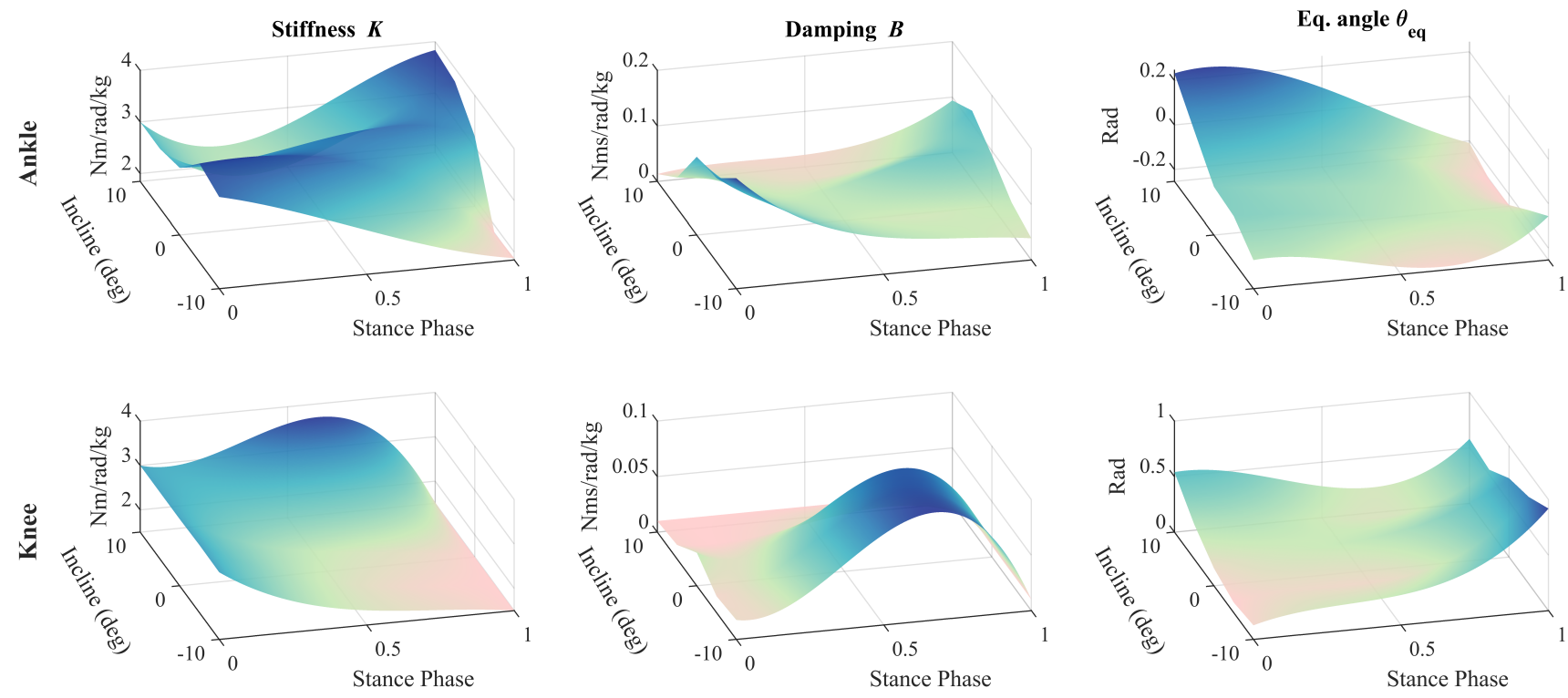

Fig. 2. Plots of the calculated impedance parameter functions, stiffness $K\left(s_{\mathrm{st}}, \gamma, \nu\right)$, damping $B\left(s_{\mathrm{st}}, \gamma, \nu\right)$, and equilibrium angle $\theta_{\mathrm{eq}}\left(s_{\mathrm{st}}, \gamma, \nu\right)$, for the knee and ankle, projected onto a speed of $\nu=1 \mathrm{~m} / \mathrm{s}$. These surfaces show the approximated solution to the original optimization problem (4).

error, detailed in the next section. Then for each task $\chi_{p}$, the inter-subject mean set of coefficients $\bar{\kappa}_{\chi_{p}}$ was calculated for use as the final model. Trials that did not well-represent the data, measured by a Variance Accounted For (VAF) below $75 \%$, were discarded as outliers prior to averaging.

\section{Modeling Results}

Fig. 2 shows the calculated impedance parameter model projected onto a speed of $1 \mathrm{~m} / \mathrm{s}$, which was produced by evaluating (2) and (3) with $\bar{\kappa}_{\chi_{p}}$. To quantify the impedance parameter model's reconstruction error, we calculated $\hat{\tau}$ for each trial in the dataset using the model:

$$
\hat{\tau}=K\left(s_{\mathrm{st}}, \gamma, \nu\right)\left(\theta_{\mathrm{eq}}\left(s_{\mathrm{st}}, \gamma, \nu\right)-\theta\right)-B\left(s_{\mathrm{st}}, \gamma, \nu\right) \dot{\theta} .
$$

Then, we calculated the root mean squared error (RMSE) in joint torque over all subjects for each task $\chi_{p}$ in the dataset and normalized by the dataset torque's standard deviation for $\chi_{p}$. This metric, which we call normalized reconstruction error $\overline{\mathrm{E}}$, describes how many standard deviations $\hat{\tau}$ is from the mean dataset torque trajectories, on average. Normalized reconstruction errors below 1.0 indicate that the model is able to predict joint torque to accuracy levels similar to ablebodied inter-subject variation [21]. Averaged over all tasks, the knee and ankle normalized reconstruction errors were $\overline{\mathrm{E}}_{\mathrm{k}}=0.78 \pm 0.11$ and $\overline{\mathrm{E}}_{\mathrm{a}}=0.58 \pm 0.09$, respectively.

\section{Hybrid Kinematic ImPedance CONTROLleR}

The proposed Hybrid Kinematic Impedance Controller (HKIC, see Fig. 1) is an evolution of the purely kinematic controller proposed in [36]. In the HKIC, we use real-time phase and task estimates with the impedance model developed in Section $\Pi$ during stance and a kinematic model developed in [20] during swing. Impedance and position controllers enforce the respective model outputs as described below. Once configured with the user's mass and leg segment lengths, the controller operates autonomously, requiring no manual tuning or terrain inputs.

\section{A. Task-Invariant Phase Estimation}

An estimate of the user's progression through the gait cycle is required to use the impedance parameter model for realtime control. An ideal version of this estimate (termed a phase variable) increases from 0 to 1 at a constant rate between each heelstrike [37]. Similar to [33], [36], the HKIC's phase variable $\hat{s}$ is calculated using a piecewise-linear mapping of the user's global thigh angle $\theta_{\text {th }}$, which has a roughly sinusoidal trajectory (see Fig. 3a). This angle is measured directly using an Inertial Measurement Unit (IMU, 3DM-CX5-25, LORD Microstrain, Williston, VT) mounted to the proximal end of the prosthesis's knee joint. This method of phase estimation is preferable because it allows the user to start and stop the gait cycle at will, is robust to sensor noise, and enables nonrhythmic behavior [33].

However, previous iterations of the $\theta_{\mathrm{th}}$-based phase variable did not work well for variable-task locomotion because of assumptions made about the shape of the $\theta_{\text {th }}$ trajectory. For example, [33], [36] assumed that the $\theta_{\text {th }}$ trajectory could be divided into two monotonic sections. While this assumption holds fairly well for level ground and incline walking, it is invalid for steep declines [21] (Fig. 3a). Previous methods produced inaccurate, saturated phase estimates for such cases [36]. Further, previous methods did not account for periods of low thigh angular velocity (i.e., when the hip joint is most extended or most flexed), leading to pauses in the phase estimate and subsequent problems in the controller behavior [24], [36]. Therefore in this work, we relax previous assumptions and add flexibility to the phase variable to better parameterize the gait cycle based on the diverse $\theta_{\text {th }}$ trajectories observed in variable-task locomotion. First, we introduce short periods of feedforward phase progression that allow $\hat{s}$ to 


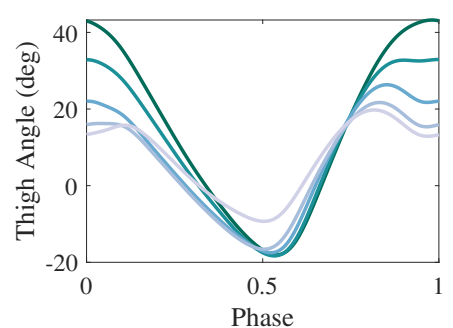

(a) Average $\theta_{\text {th }}$ trajectory from $[21]$

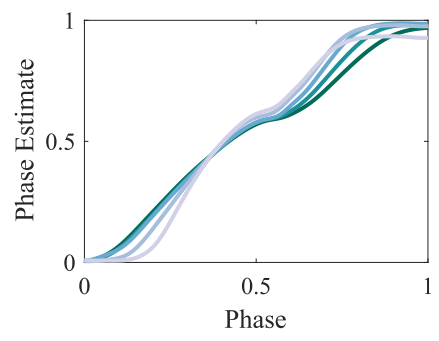

(b) Phase variable from 36

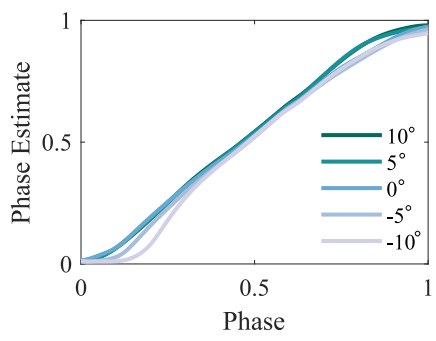

(c) Phase variable from this work

Fig. 3. Plots of (a) the mean able-bodied thigh trajectories reported in [21], where positive angles correspond to hip joint flexion, and (b)-(c) the resulting phase variable trajectories at different inclines. Plot (b) shows the trajectories calculated the previous method described in [36] and Plot (c) shows the trajectories calculated using the new phase variable proposed in this work. The new method shows no phase pause near push-off and improved linearity, especially at the point of maximum hip extension.

maintain a constant positive rate even when thigh angular velocity is low, which enables a biomimetic, powerful pushoff. Second, we add states to account for thigh trajectories that have more than two monotonic sections (especially common during ramp descent) to prevent phase saturation and resulting gait desynchronization. Third, we introduce a technique to improve the linearity of $\hat{s}$, correcting for previous steadystate nonlinearities and thus making it closer to an ideal phase estimate. For brevity, the mathematical details for these improvements are presented in Appendix A

To illustrate the benefits of the new phase variable over its predecessor [33], [36], we conducted a simulation using thigh kinematic data from [21] (Fig. 3a). For each trial of treadmill walking in the dataset, we calculated the phase variable using both the new method (Appendix A and the previous method described in [36]. For each incline, we averaged the phase trajectories over all strides, participants, and walking speeds, shown in Figs. $3 b$ and $3 \mathrm{c}$ Notably, the new phase variable eliminated the phase estimate pause associated with maximum hip extension that was observed with the previous phase variable. The new method also reduced the early saturation seen in the previous phase variable, which was particularly prominent at steep ramp declines. Finally, the new method demonstrated improved linearity, particularly during midstance. Compared to an ideal linear phase trajectory, the new method showed 4.50\% RMSE with $R^{2}=0.991$ while the previous method showed $7.48 \%$ RMSE and $R^{2}=0.976$ over all tasks.

\section{B. Task Estimation}

In addition to the phase estimate, the HKIC requires an estimate of the user's current task $\hat{\chi}$, which is calculated at each TO during steady walking. The estimation methods below are based on [36], with modifications to improve performance. Both estimates are filtered with a moving average over 3 strides to account for stride-to-stride variation.

1) Walking Speed: The user's speed is estimated using a three-link leg model, comprising thigh, shank, and foot links. Using forward kinematics and inputs from the joint encoders and the thigh IMU, we calculate the Cartesian locations of the heel and toe relative to the hip joint, respectively given by $x_{\text {heel }}$ and $x_{\text {toe }}$. At each TO event, the forward progression of the hip relative to the foot's point of contact with the ground during the previous stance phase is calculated as

$$
d_{\mathrm{st}}=\left\|x_{\text {toe }}-x_{\text {heel }}^{\mathrm{HS}-}\right\|_{2},
$$

where $x_{\text {heel }}^{\mathrm{HS}-}$ is the value of $x_{\text {heel }}$ from the previous HS. Similarly by assuming a symmetric gait, the forward progression of the hip relative to the contrallateral foot's ground contact point over a swing phase is approximated at each HS as

$$
d_{\text {sw }}=\left\|x_{\text {heel }}-x_{\text {toe }}^{\mathrm{TO}-}\right\|_{2},
$$

where $x_{\text {toe }}^{\mathrm{TO}-}$ likewise is $x_{\mathrm{toe}}$ from the previous TO. Then, we calculate the total forward progression over the gait cycle as $d_{\mathrm{st}}+d_{\mathrm{sw}}+\ell_{\text {foot }}$, where $\ell_{\text {foot }}$ is a constant accounting for the length of the prosthetic foot. Finally, walking speed is estimated by dividing forward progression by stride time.

2) Incline: The global angle of the foot, $\theta_{\mathrm{f}}$, is a good approximation for the incline when the foot is flat on the ground. As it is undesirable to add an inertial sensor to the foot, we calculate this angle from the thigh IMU using forward kinematics, along with a correction for foot bending. Prosthetic feet are designed to deflect for energy storage [38], so foot deflection significantly impacts the incline estimate. Offline testing with our prosthesis's foot [39] (Lo Rider, 1E57, Ottobock, Duderstadt, Germany) showed that deflection was correlated with the bending moment in the sagittal plane $m_{y}$. An on-board 6-axis load cell (M3564F, Sunrise Instruments, Nanning, China), located at the distal end of the ankle joint, measures this moment directly. Then, $\theta_{\mathrm{f}}$ is calculated as

$$
\theta_{\mathrm{f}}=\theta_{\mathrm{th}}-\theta_{\mathrm{k}}+\theta_{\mathrm{a}}+\theta_{\mathrm{f}}^{0}+k_{f} m_{y},
$$

where $k_{f}$ is the linear bending coefficient, $\theta_{\mathrm{k}}$ is the relative knee angle, and $\theta_{\mathrm{a}}$ is the relative ankle angle. All joint angles are measured positive in flexion and are zero when the user stands upright. The constant offset term $\theta_{\mathrm{f}}^{0}$ accounts for the angular difference between the prosthetic foot, the cosmesis, and the sole of the shoe.

The center of pressure in the foot reference frame $\ell_{\text {cop }}$ is calculated using the load cell to determine when the foot was flat on the ground. We consider the foot to be flat when $7.5 \leq$ $\ell_{\text {cop }} \leq 12 \mathrm{~cm}$ from the ankle joint, which corresponds to the ground reaction force acting between the middle and the ball of the foot. During this period, $\theta_{\mathrm{f}}$ is averaged to produce the incline estimate for each stride. 


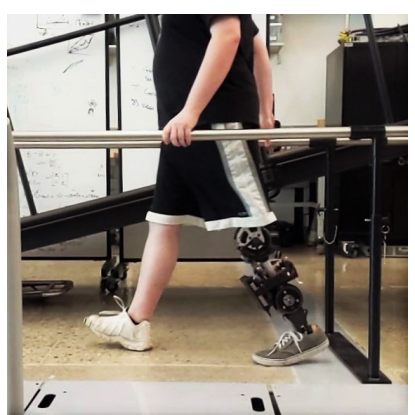

(a) Overground acclimation

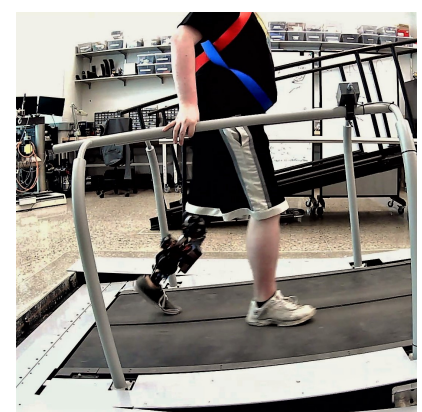

(b) SS-Incline: $\chi=7 \mathrm{deg}, 1 \mathrm{~m} / \mathrm{s}$

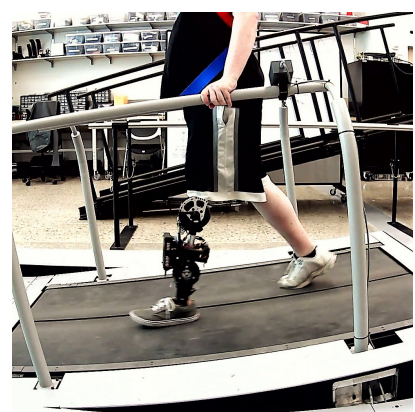

(c) SS-Decline: $\chi=-7 \mathrm{deg}, 1 \mathrm{~m} / \mathrm{s}$

Fig. 4. Photos of the participant with an above-knee amputation performing various tasks with the HKIC during the experiment.

\section{Impedance and Kinematic Controllers}

1) Stance Impedance Controller: During stance, a variable impedance controller is used to calculate joint torques. First, the stance phase estimate $\hat{s}_{\mathrm{st}}$ is calculated by

$$
\hat{s}_{\mathrm{st}}=\hat{s} / \overline{\hat{s}}_{\mathrm{TO}},
$$

where $\overline{\hat{s}}_{\mathrm{TO}}$ is a low pass filtered (IIR) version of $\hat{s}_{\mathrm{TO}}$ from previous strides. A slow time constant of approximately 10 strides was chosen for this filter, as the toe-off phase exhibits slow changes with task. Like the thigh trajectory features, the filter for $\overline{\hat{s}}_{\text {TO }}$ is initialized from able-bodied data. Using $\hat{s}_{\text {st }}$ and $\hat{\chi}$, joint stiffness $K$, damping $b$, and equilibrium angle $\theta_{\mathrm{eq}, \chi_{p}}$ are calculated using (2), 3) and the model in Section [I] Then, the joint torque for stance is calculated with the following impedance control law (scaled by user mass $m$ ):

$$
\tau_{\mathrm{st}}=m\left(K\left(\hat{s}_{\mathrm{st}}, \hat{\chi}\right)\left(\theta_{\mathrm{eq}, \chi_{p}}\left(\hat{s}_{\mathrm{st}}, \hat{\chi}\right)-\theta\right)-B\left(\hat{s}_{\mathrm{st}}, \hat{\chi}\right) \dot{\theta}\right) .
$$

2) Swing Kinematic Controller: A proportional derivative (PD) controller uses stiff gains $k_{\mathrm{p}}$ and $k_{\mathrm{d}}$ to directly track desired joint angle trajectories. This is in contrast to the equilibrium angles of the impedance controller, which do not necessarily align with the normative joint angles. A continuous model of able-bodied joint kinematics [20], generated using data from [21], provides the desired trajectories defined as

$$
\theta_{\mathrm{d}}(s, \chi)=\sum_{i=1}^{N} b_{k}(s) c_{k}(\chi),
$$

where $b_{k}(s)$ are Fourier series and $c_{k}(\chi)$ are Bernstein basis polynomials. Similar to the impedance model, (19) is evaluated in real-time using $\hat{s}$ and $\hat{\chi}$. Then, the PD torque command during swing, $\tau_{\mathrm{sw}}$, is given by

$$
\tau_{\mathrm{sw}}=k_{\mathrm{p}}\left(\theta_{\mathrm{d}}-\theta\right)+k_{\mathrm{d}}\left(\dot{\theta}_{\mathrm{d}}-\dot{\theta}\right) .
$$

3) Stance to Swing Transition Smoothing: A time-varying weight $w_{\mathrm{sw}}$ ensures a smooth transition from impedance control to position control. Because impedance control may allow the joint angles to vary from their nominal trajectories depending on how the user loads the prosthesis, this smoothing is critical to avoid step changes in joint torque. At the instant of the stance to swing transition, $w_{\mathrm{sw}}$ increases from 0 to 1 over a predefined time frame. For the knee, it increases over
$0.25 \mathrm{~s}$ and for the ankle over $0.05 \mathrm{~s}$. The actual output to the joint motors is given by

$$
\tau= \begin{cases}\tau_{\mathrm{st}} & \text { during Stance, } \\ w_{\mathrm{sw}} \tau_{\mathrm{sw}} & \text { during Swing. }\end{cases}
$$

Because the equilibrium angles at heelstrike are close to the kinematic references at the end of the gait cycle, no smoothing is necessary for the swing to stance transition.

Close examination of 21) shows that for a brief period just following TO, minimal control action is applied to the joints. This is acceptable because the low-impedance actuators used in our prosthesis [39] allow the joints to continue moving along their current trajectories according to their passive dynamics without control input. Passive early swing knee and ankle dynamics have been shown to produce human-like gait [40], [41], and these passive dynamics may contribute to the biomimetic behavior of the controller.

\section{Amputee Participant Experiment}

Experiments with an above-knee amputee participant were performed to investigate the benefits of the continuouslyvarying framework and the effectiveness of the optimizationderived impedance parameters at producing a biomimetic gait. For comparison, we implemented and tuned a standard, piecewise-constant impedance controller based on a FSM. This type of FSM controller has been widely implemented as a standard, effective method of impedance control for walking applications [1], and therefore provides a good benchmark with which to compare our controller. Photos of the experiment are shown in Fig. 4 and video recordings are available for download as supplemental media.

\section{A. Benchmark FSM Impedance Controller}

A benchmark Finite State Machine controller (FSMC) was designed based on the FSM impedance controller presented in [15], with an additional stance state and modified transition criteria to improve performance (see Appendix B]. The resulting FSM had 5 states, each with its own set of constant impedance parameters and transition criteria. Similar to the methods discussed in the introduction, these parameters needed to be hand-tuned by an expert researcher in order to produce the desired gait. To enable walking at various inclines, three sets of tunable impedance parameters and transition 


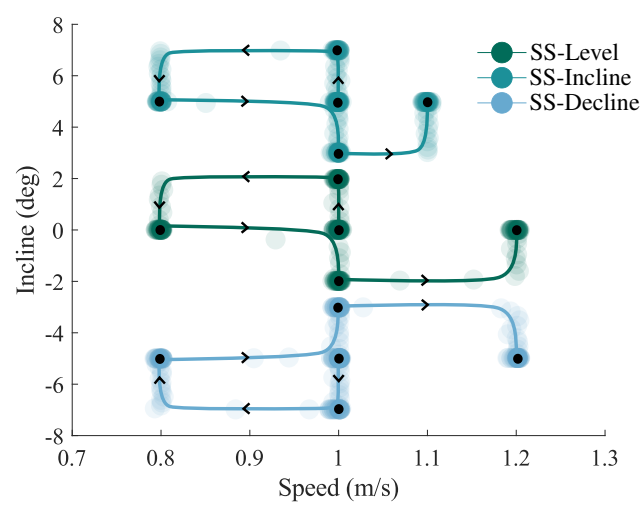

(a) Steady-state task trials

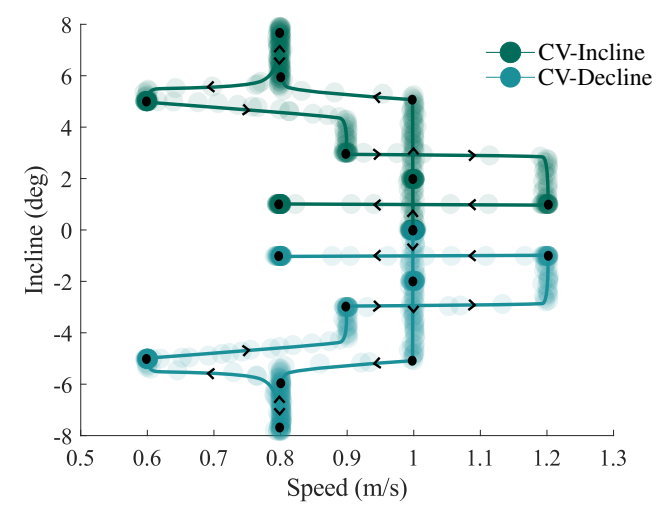

(b) Continuously-varying task trials

Fig. 5. Diagrams indicating the locations of the task space sampled during each trial. Each transparent marker indicates the treadmill's task feedback, sampled at $2 \mathrm{~Hz}$. Each black dot indicates the task combination commanded to the treadmill for a duration of 45 seconds in (a) and 20 seconds in (b).

criteria were instantiated for each joint (i.e., one set for level ground, one set for declines, and one set for inclines). The controller selected between impedance parameter sets based on the estimated incline $\hat{\gamma}$ (Appendix Fig. 13b). In total, the FSMC required 96 tunable parameters, including 45 impedance parameters per joint and 6 FSM transition criteria.

\section{B. Experimental Methods}

A participant with an above-knee amputation (sex: male, age: 26 years, mass: $116 \mathrm{~kg}$, height: $1.9 \mathrm{~m}$, K-level: 4 , cause of amputation: congenital, time since amputation: 26 years) was recruited to participate in the experiment. The protocol was approved by the Institutional Review Board of the University of Michigan (HUM00166976) and the participant wore a ceiling-mounted safety harness while walking on the treadmill.

The proposed HKIC and the comparison FSMC were implemented on a backdrivable, powered knee-ankle prosthesis, shown in Fig. 4 and described in depth in [39]. A licensed prosthetist fit the prosthesis to the participant and ensured proper alignment. The participant was instructed on the expected high-level behavior of each controller and given time to acclimate to each while walking overground within parallel bars. Following this overground acclimation, 5 trials with each controller were conducted on an in-ground split-belt treadmill (Bertec, Columbus, Ohio, USA). The treadmill had instrumented handrails on either side for safety, and the participant was instructed to limit handrail body weight support when possible to maximize the realism of the experiment. The instrumented handrail data showed that the subject did not heavily rely on the handrails with either controller.

The first 3 trials investigated the behavior differences between the HKIC and the FSMC during steady walking at different speed and incline combinations. Each trial focused on a range of small task deviations ( $\pm 2 \mathrm{deg}, \pm 0.2 \mathrm{~m} / \mathrm{s}$ ) around one of three baseline tasks: $\chi=(0 \mathrm{deg}, 1 \mathrm{~m} / \mathrm{s}), \chi=(5 \mathrm{deg}$, $1 \mathrm{~m} / \mathrm{s})$, and $\chi=(-5 \mathrm{deg}, 1 \mathrm{~m} / \mathrm{s})$. We refer to these steadystate task trials as SS-Level, SS-Incline, and SS-Decline, respectively. For the SS-Incline trial, speed was limited to 1.1 $\mathrm{m} / \mathrm{s}$ to ensure that the participant could safely perform the trial. The steady-state task trials began with an acclimation period, where the participant walked at the baseline task until feeling comfortable. During this time, the FSMC was tuned by the research team to produce a natural gait, incorporating feedback from the participant and the prosthetist. The time taken to tune the controller was recorded. Note that no tuning was done for the HKIC. Then, the participant walked on the treadmill as it cycled through each of the 5 tasks within the small range, each commanded for 45 seconds. The acclimation, tuning, and testing procedure above was repeated for each baseline task. These baseline tasks were chosen to be far apart in the task space so that we could sample a wide range of tasks without deviating too far from any one of the FSMC's tuning points. Fig. 5a shows the recorded task-space profiles from the treadmill for each trial, where the black dots indicate each commanded task. Note that both controllers were provided with true task feedback from the treadmill during these trials so that task estimate errors did not influence the results.

The latter two trials consisted of more rapid task changes to investigate each controller's behavior during continuous task variations rather than at steady-state and over a wider range of tasks. Further, the controllers received no real-time knowledge of the task from the treadmill during these trials, investigating the autonomous capability of each controller to operate over the task space. Both controllers utilized the same task estimation methods (Section III-B). The FSMC used the task transition logic in Figure 13b with the tuned impedance parameters at the baseline tasks from the steady-state trials. Two trials, one with inclines (CV-Incline) and the other with declines (CV-Decline), started at $\chi=(0 \mathrm{deg}, 1 \mathrm{~m} / \mathrm{s})$ and explored 8 other points within the task space. Each task point was commanded to the treadmill for 20 seconds. Because the treadmill required time to change task, smooth task trajectories with continuous variations were generated, shown in Fig. $5 b$.

\section{Experimental Results}

1) FSMC Tuning Time: To produce a normal gait, the FSMC required 22 minutes of tuning by the research team: 3 minutes for the level ground baseline task, 14 for the incline baseline task, and 5 for the decline baseline task. The tuned impedance and state transition parameters for each baseline 

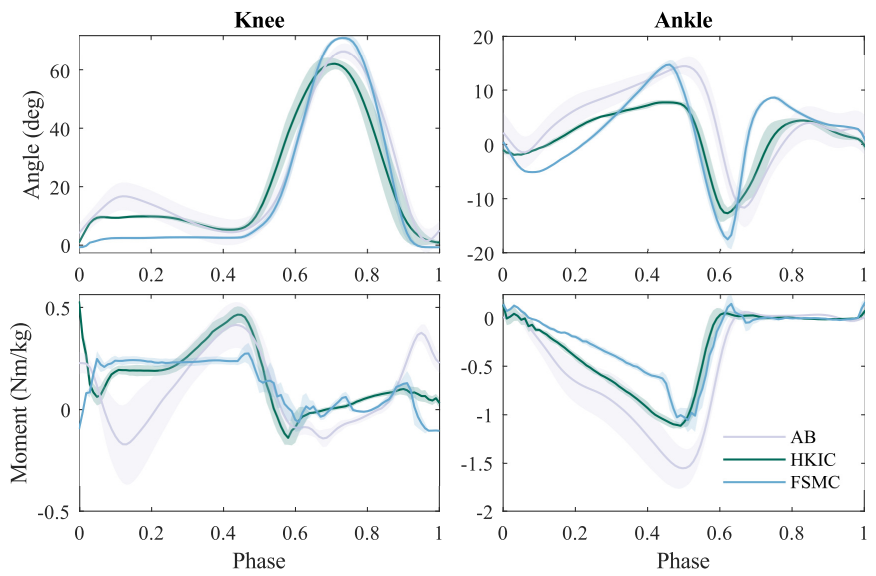

Fig. 6. Average kinematic and kinetic trajectories produced by each controller during level ground walking at $1 \mathrm{~m} / \mathrm{s}$ in the steady-state trials. Inter-subject mean able-bodied data (AB) is also shown [21]. Shaded regions show \pm 1 standard deviation. At most points in the gait cycle, the HKIC is closer to the able-bodied reference than the FSMC, particularly in the kinetic trajectories.

task are shown in the Appendix (Table IV). Trends in the tuned parameters included higher stiffness values during stance than in swing and highly-varying knee equilibrium angles across tasks. Also, the observed gait was noted to be quite sensitive to the tunable FSM transition criteria.

2) Steady-State Trials: The steady-state trials were examined for kinematic and kinetic similarity relative to able-bodied data [21]. Bilinear interpolation was used to generate the ablebodied data for tasks between those reported in the dataset. Fig. 6 shows the kinetic and kinematic trajectories for the level ground, $1 \mathrm{~m} / \mathrm{s}$ trial for each controller and the ablebodied references. The HKIC is closer to the able-bodied references than the FSMC at most points in the gait cycle. Additionally, discontinuities caused by the FSMC's discrete FSM states transitions can be seen in the figure, particularly in the ankle moment near midstance. Next, the RMSE between the observed data and able-bodied data was calculated during both stance and swing for all strides in the steady-state task trials. Stance and swing were treated separately to isolate the performance of the impedance parameter model (Section III), as it was only used during stance. The first 15 seconds at each task was neglected to allow time for the treadmill to reach steady-state. Fig. 7 shows the mean kinetic and kinematic RMSE averaged over all strides for the steady-state trials. In 7 of 8 comparisons, the HKIC showed less error than the FSMC. Only the knee kinematic error during swing showed less error with the FSMC than the HKIC.

The gait energetics were also examined for able-bodied similarity. As one of the benefits of impedance control is the ability to control energy exchange with the environment [9], both controllers should replicate this biological behavior. Fig. 8 shows the average knee, ankle, and combined net work per stride performed by each controller at different inclines during the steady-state trials, as well as average able-bodied data calculated from [21]. The HKIC shows similar trends as the able-bodied data, with a linear increase in net energy input with incline, particularly at the ankle (increase of 3.81 $\left.\mathrm{J} / \mathrm{kg} / \mathrm{deg}, R^{2}=0.995\right)$. For comparison, able-bodied ankle work increases linearly at $4.06 \mathrm{~J} / \mathrm{kg} / \mathrm{deg}$ with $R^{2}=0.987$. In
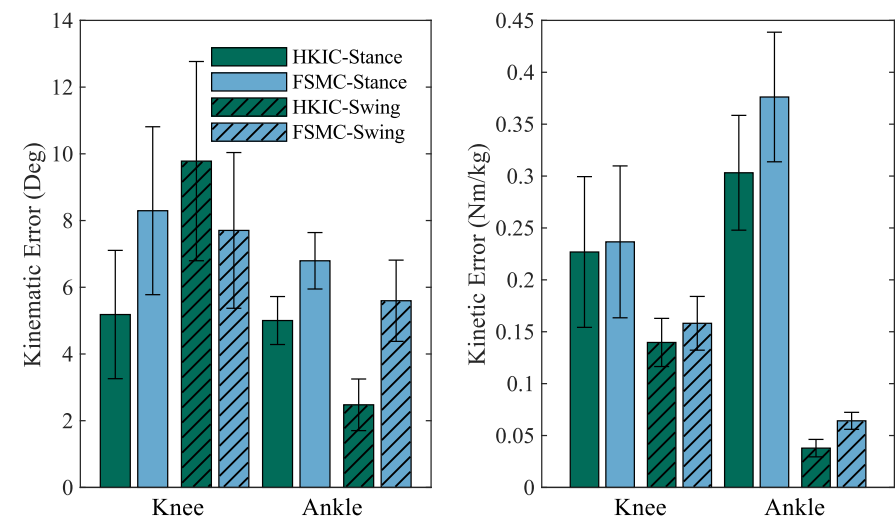

Fig. 7. Mean RMSE in the observed kinematics (left) and kinetics (right) relative to able-bodied walking data for both the HKIC and FSMC during the steady-state task trials. The error bars represent \pm 1 standard deviation. The HKIC demonstrated lower mean error than the FSMC in 7 of 8 metrics.

TABLE I

AVERAGE KINEMATIC AND KINETIC RMSE OBSERVED DURING THE CONTINUOUSLY-VARYING TASK TRIALS

\begin{tabular}{|c|c|cccc|}
\hline \multirow{2}{*}{ Trial } & \multirow{2}{*}{ Controller } & \multicolumn{2}{|c|}{ Angle (deg) } & \multicolumn{2}{c|}{ Torque } \\
\cline { 3 - 6 } & & Knmee & Ankle & Knee & Ankle \\
\hline \multirow{2}{*}{ CV-Incline } & HKIC & 6.13 & 4.59 & 0.15 & 0.22 \\
& FSMC & 9.35 & 8.77 & 0.21 & 0.35 \\
& HKIC & 7.34 & 5.17 & 0.21 & 0.27 \\
CV-Decline & FSMC & 8.46 & 6.57 & 0.19 & 0.35 \\
& & & & &
\end{tabular}

contrast, the energy injection of the FSMC appears discretized to three levels corresponding to its tuning tasks. Interestingly, the HKIC and FSMC show less energy absorption at the knee during declines, which may indicate a compensatory behavior by the participant.

3) Continuously-Varying Trials: The kinematic and kinetic errors were calculated in a similar manner for the continuously-varying task trials, though this time including strides that occurred while the treadmill changed task. For brevity, the error metrics were calculated over the entire stride (instead of separating stance and swing) and averaged across all strides of the trial, shown in Table [1] The PV controller showed lower RMSE than the FSM controller in all metrics for the CV-Incline trial and 7 of 8 metrics for the CVDecline trial. In addition, Fig. 9 shows the average error trajectories at both joints for the CV-Incline trial, calculated as the able-bodied references subtracted from the observed values. At most points in the gait cycle, the FSMC showed higher magnitude kinematic and kinetic error than the HKIC, particularly in the ankle kinematics near push-off.

The task estimates and HKIC phase estimates contributed to the kinematic and kinetic errors in these trials. Fig. 10 shows the average phase estimate trajectories produced by HKIC during the CV-Incline and CV-Decline trials. The average phase trajectories were highly linear, even as speed and incline varied. Also, the task estimate RMSE, averaged over each stride, is shown in Table II Although the same task estimation algorithms were used with both controllers, FSMC showed higher incline estimate error, suggesting that differences in controller behavior impacted the incline estimate's efficacy. 

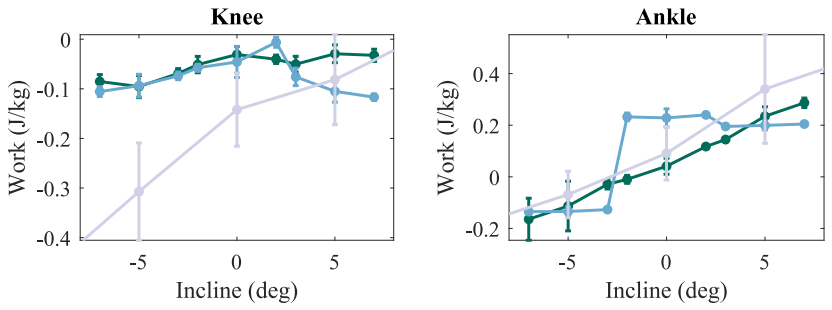

Total

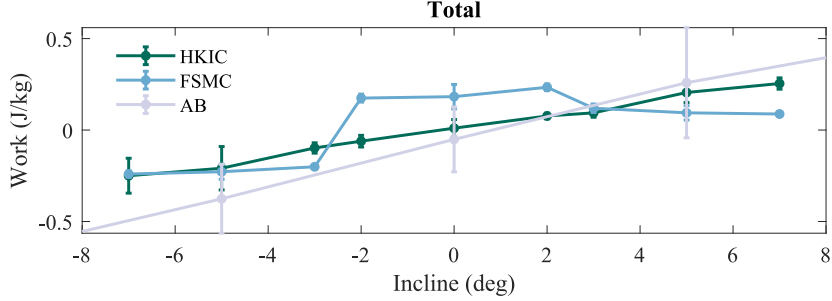

Fig. 8. Average net work per stride performed by the prosthesis at each of the inclines tested during the steady-state task trials. Error bars represent \pm 1 standard deviation. An able-bodied reference (AB) calculated from [21] shows that the HKIC demonstrated more biomimetic energy injection, particularly through a linear increase in ankle work as incline increased, corresponding to $93.8 \%$ of the able-bodied rate. Both controllers showed less energy absorption at the knee during steep declines, suggesting that our participant may have had a habitual aversion to early stance knee flexion.

TABLE II

AVERAGE TASK ESTIMATE RMSE OBSERVED DURING THE CONTINUOUSLY-VARYING TASK TRIALS

\begin{tabular}{|c|c|c|c|}
\hline Trial & Controller & Incline $(\mathbf{d e g})$ & Speed $(\mathbf{m} / \mathbf{s})$ \\
\hline \multirow{2}{*}{ CV-Incline } & HKIC & 0.76 & 0.15 \\
& FSMC & 3.04 & 0.15 \\
& HKIC & 0.67 & 0.15 \\
\multirow{2}{*}{ CV-Decline } & FSMC & 1.52 & 0.12 \\
\hline
\end{tabular}

\section{Discussion}

This work presented a data-driven, phase-based walking controller for a powered knee-ankle prosthesis that autonomously adapted its behavior across a continuous range of walking speeds and inclines. To achieve this without manual tuning, we used an able-bodied dataset to optimize for continuous stiffness, damping, and equilibrium angle functions that reproduced biological joint torques during stance, given biological kinematics. We showed that our optimized impedance parameter model produced joint torques with across-task average normalized RMSE values of 0.78 and 0.58 for the knee and ankle, suggesting that the model captures the essential joint dynamics of able-bodied walking.

The subsequent experiment demonstrated that the identified impedance parameter functions also rendered appropriate stance phase joint mechanics for a participant with an aboveknee amputation. The participant exhibited qualitatively normal gait patterns over a wide array of tasks (see Supplemental Video). Other normative walking features were also observed, such as increased ankle work with increasing incline. Anecdotally, our participant remarked while walking at the $7 \mathrm{deg}$ incline that he did not feel like he was walking uphill, suggesting appropriate joint dynamics and energy exchange. Further, HKIC's kinematic and kinetic errors during stance were better than those produced using a hand-tuned impedance controller (Fig. 7). Together, these results suggest that the data-driven
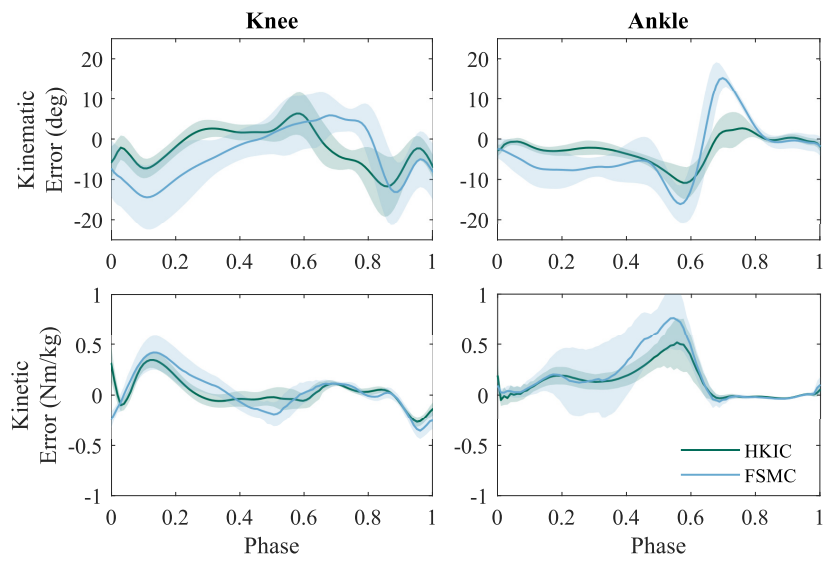

Fig. 9. Plot of the average kinematic and kinetic differences relative to ablebodied observed in the continuously-varying incline trial. The knee data is shown in the left column and the ankle in the right. Shaded regions represent \pm 1 standard deviation. For this trial, the HKIC demonstrated lower RMSE compared to the FSMC in all 4 metrics, suggesting that it behaved more biomimetically across the incline and speed variations.

approach to calculating impedance parameters was effective at producing appropriate joint mechanics across the task-space, presenting an attractive alternative to the standard hand-tuning paradigm typically required with impedance controllers.

For online implementation of the continuous impedance parameter model, gait phase needed to be estimated in real-time. We employed a novel phase variable based on the user's thigh angle that improved on previous limitations associated with a $\theta_{\text {th }}$-based approach. The improved phase variable behavior observed in simulation in Section III-A was confirmed in the participant experiment. Fig. 10 shows how the phase variable eliminated the previously observed phase pause near push-off. The result of this monotonicity is visible in the kinematics of Fig. 9, as there is not a sharp increase in kinematic error near push-off, which was observed previously in [36]. Further, minimal saturation is observed near the end of the gait cycle in both continuously-varying trials. As the CV-Decline trial contained strides at steep declines, previous iterations of the phase variable would have resulted in early saturation, as the maximum $\theta_{\mathrm{th}}$ is much larger than $\theta_{\mathrm{th}}$ at HS. The lack of phase saturation illustrates that the new phase variable effectively handled task-varying $\theta_{\mathrm{th}}$ trajectories. Finally, the general linearity of the average phase trajectories in Fig. 10 (mean RMSE of $3.33 \%$ and $R^{2}=0.997$ with respect to an ideal linear trajectory) is improved compared to [36]. Because both the impedance and kinematic models in HKIC assume a perfectly linear phase estimate, the observed linearity keeps the model outputs of the controller synchronized with the user's gait.

The task estimates are other critical components required for walking over continuously-varying tasks. Seen in Table [II. the error in the speed estimate was fairly constant over the trials, with RMSE between 0.12 and $0.15 \mathrm{~m} / \mathrm{s}$ for both controllers. This error is likely due to a slightly asymmetric gait, which violates the assumptions made in the speed estimator's formulation. Gait asymmetries may be the result of our participant's habitual compensations, socket comfort, or the significant mass difference between the robotic prosthesis 

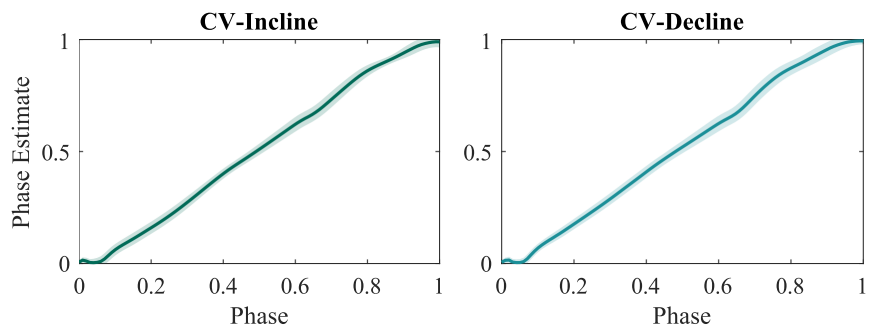

Fig. 10. Average phase estimate progression calculated in real-time by the HKIC during the continuously-varying task trials. Shaded regions represent \pm 1 standard deviation. The linearity and consistency of the trajectories illustrate the phase variable's ability to adapt to continuous task variations and appropriately parameterize the gait cycle.

and participant's passive prosthesis. Interestingly, the incline estimate produced lower error with the HKIC (0.67 to 0.76 deg) than the FSMC (1.52 to $3.04 \mathrm{deg}$ ) in both trials. We speculate that the higher error in the FSMC is due to a feedback interaction between incline estimate errors and the impedance parameters. Due to the discrete switching behavior of the impedance parameters (see Fig. 13b), a small incline estimate error can result in large changes in prosthesis behavior. If this change is inappropriate for the current task, such as when $\gamma=1.5$ but $\hat{\gamma}=3.5$, the resulting progressions of $\theta_{\mathrm{f}}$ and $\ell_{\text {cop }}$ may be atypical and further skew the incline estimate. Therefore, the continuous nature of the HKIC is preferable, as it does not display step changes in behavior with small changes in task inputs.

Experimental validation demonstrated that, overall, the HKIC was able to better reproduce normative joint biomechanics than the FSMC, despite requiring no subject-specific tuning. During the steady-state trials, which tested tasks at or near the tuned tasks of the FSMC, the HKIC resulted in lower kinematic and kinetic error relative to able-bodied data for all metrics at the ankle and most metrics at the knee. The only metric in which the FSMC outperformed the HKIC was the knee kinematic error in swing. This was expected, as we intentionally allowed the phase variable to saturate early and, as the knee displays large kinematic changes during swing, small phase shifts result in large kinematic errors. We made this tradeoff to ensure full knee extension prior to heelstrike, as pilot testing showed that consistent full knee extension helped eliminate participants' problematic instinctive compensations (see Section [III-A). Although the knee kinematic error values appear relatively large, it did not interfere with the participant's gait or cause toe stubbing. In comparison, the FSMC required 22 overall minutes of tuning for just three tasks, and it displayed overall lower performance than the HKIC. Its performance likely could have been improved to better mimic normative data, but this would have required either more tuning time at each baseline task or additional tuning points throughout the task space. Adding tuning time or points is likely impractical in a clinical setting, especially without specialized equipment such as a variable-incline treadmill. Therefore, a tuning-free approach like the HKIC is advantageous because it produces biomimetic behavior regardless of task, and it is quickly configured with only physical measurements of the user.
Further differences between the HKIC and the FSMC were illustrated in the continuously-varying task trials, in which the two controllers operated fully autonomously. While it is difficult to isolate the influence of task estimate errors on the resulting kinematics and kinetics, some comparisons can still be made. For example, the FSMC's large sinusoidalshaped ankle kinematic error just after push-off (Fig. 9) is indicative of a phase shift between the observed and ablebodied kinematics, suggesting improper gait cycle timing. The kinematic error after push-off was lower for the HKIC, suggesting that the new phase variable was better at adapting gait cycle timing than the FSMC transition criteria. In addition, the FSMC produced a larger standard deviation in both joints' kinematic error trajectories and the ankle's kinetic error. This suggests that the FSMC produced less consistent mechanics stride-to-stride than the HKIC, which is likely due to its discrete switching nature. The more consistent behavior of the HKIC is desirable because it allows the user to better predict the prosthesis's behavior and walk more confidently.

However, this study was not without limitations. Our experiment provided a somewhat limited view of the HKIC's behavior, as it involved a single participant and only one experimental session. We expect that the tuning-free impedance parameter model identified in Section [II will yield similar performance for a wide array of participants, as it was created without a priori knowledge of the participant or their preferences. Preliminary studies of able-bodied users testing the HKIC over varying tasks suggest that this assumption holds [42]. However, this assumption should be validated in future studies with wider prosthesis user participant pools.

Similarly, it is likely that there are users for which the population average impedance parameters are not optimal. A study investigating users' preferred stiffness in ankle prostheses showed that the preferred joint stiffness varies by user [43], which is likely true for knee-ankle prosthesis users as well. While one of the major advantages of HKIC is that it required no manual tuning, it could be limited by the lack of an ability to customize to an individual's preferred behavior. Future work will investigate methods to incorporate user preferences in the impedance model, such as weighting the optimization with a single baseline personalization for level-ground walking, as suggested in [44]. This baseline personalization could be gathered using tools in a standard clinic, maintaining the minimal-tuning nature of the controller.

Further, future studies investigating the HKIC's performance over multiple experimental sessions would be beneficial because they would allow the participant to better acclimate to and leverage the benefits the powered prosthesis. For example, the lack of early stance knee flexion in both controllers (Fig. 9) and the low knee energy dissipation (Fig. 8) are likely less due to controller behavior and more due to the participant's habitual compensations developed through years of using a passive prosthesis [45]. Future work may show that as the participant becomes more comfortable with a powered prosthesis, these gait features become more similar to ablebodied gait, even with the same controller.

In addition, this work did not fully explore the capabilities of the HKIC, as it only investigated rhythmic walking. One of the 
unique strengths of the proposed phase variable is the ability to intuitively control non-rhythmic tasks [33]. This capability of the HKIC should be explored further and demonstrated in future studies involving rapid start/stop, lateral movements, and other behaviors that are prominent in agile locomotion. Such studies may also highlight the limitations of using the current impedance parameter model for non-rhythmic tasks. Although we were able to achieve start/stop behaviors in this experiment, additional able-bodied data may need to be included in the optimization to produce appropriate impedance parameters for other non-rhythmic tasks.

Finally, there is much interesting work to be done investigating the relationship between biological joint impedance measured in empirical studies [11]-[13] and the impedance parameters used in impedance controllers. Mechanical impedance can only be characterized through perturbation studies, so the impedance parameters found by optimizing over non-perturbed gait data will not necessarily reflect these dynamics. We plan to study the effects of constraining the optimization with known empirical impedance values, as well as to investigate the HKIC's behavior during gait perturbations.

\section{CONCLUSION}

This work proposed a tuning-free walking controller designed to work over a continuum of speeds and inclines. We developed continuous models of joint stiffness, damping, and equilibrium angle for an impedance controller using a data-driven approach. We also presented an improved phase estimation algorithm, showing increased monotonicity and linearity. A prosthesis user demonstrated the controller's ability to autonomously produce biomimetic behavior over continuously-varying tasks during treadmill experiments. The experiments showed that, when compared with able-bodied data, the proposed controller performed as well as a handtuned FSM impedance controller near the FSM controller's tuning points, and had superior performance as task varied.

\section{APPENDIX}

\section{A. Task-Invariant Phase Variable Algorithm}

The new phase variable $\hat{s}$ is calculated through a series of linear equations with $\theta_{\text {th }}$ as an input. An FSM controls when each equation is used. Although the FSM contains discrete states, the structure of the linear equations ensures that $\hat{s}$ is continuous. Each equation is defined by quantitative features of the $\theta_{\text {th }}$ trajectory, which are measured in real-time. Table III lists the features' definitions and notations. First, we give the rationale for each FSM state and its corresponding phase variable equation. Then, we present methods to estimate the thigh trajectory features in real-time, as well as the steps taken to promote closed-loop stability of the phase estimate.

1) Phase Variable FSM: Consider the average $\theta_{\text {th }}$ trajectory for an able-bodied individual walking at $1 \mathrm{~m} / \mathrm{s}$ on level ground, shown in Fig. 11. The pertinent $\theta_{\text {th }}$ trajectory features used in the phase estimate are labeled, as well as the standard timing of the FSM states. The overall structure of the FSM used to control the phase estimate is shown in Fig. 12.
The FSM begins in S1, occurring just after a heelstrike (HS) event. During $\mathrm{S} 1, \theta_{\text {th }}$ is linearly scaled as the hip joint extends from $\theta_{\text {th }}^{\text {HS }}$ to $\theta_{\text {th }}^{\text {MHE }}$ such that $\hat{s}$ increases and $\hat{s}=s_{\text {MHE }}$ when $\theta_{\mathrm{th}}=\theta_{\mathrm{th}}^{\mathrm{MHE}}$. Mathematically, this is given by

$$
\hat{s}=\frac{\theta_{\mathrm{th}}^{\mathrm{HS}}-\theta_{\mathrm{th}}}{\theta_{\mathrm{th}}^{\mathrm{HS}}-\theta_{\mathrm{th}}^{\mathrm{MHE}}} s_{\mathrm{MHE}} \quad \text { in } \mathrm{S} 1, \mathrm{~S} 2 .
$$

The FSM transitions to S2 at a phase estimate threshold $\hat{s}_{1 \rightarrow 2}=0.1$, which typically corresponds to the point in the gait cycle where the $\theta_{\text {th }}$ trajectory becomes linear.

In S2, $\hat{s}$ is calculated using the same linear relationship as in S1 (22), but is denoted as a distinct state because it represents a portion of the gait cycle where $\theta_{\text {th }}$ (and therefore $\hat{s}$ ) has constant velocity. The average rate of change of $\hat{s}$ during S2 $\left(\dot{\hat{s}}_{S 2}\right)$ is recorded for use in S3. The FSM transitions to S3 once $\hat{s}_{2 \rightarrow 3}=0.9 s_{\mathrm{MHE}}$, which typically corresponds to the end of the linear portion of the thigh trajectory, or if $\dot{\theta}_{\text {th }}>0$. This second case rarely occurs during steady walking, but is an important path to S3 in the event of an unusually short stride.

S3 occurs during the section of the gait cycle where $\theta_{\text {th }}$ reaches its minimum, and thus has a period of low angular velocity $\dot{\theta}_{\text {th }}$. Previous work has shown that sections of low $\dot{\theta}_{\text {th }}$ are problematic because they cause a pause in the phase variable trajectory [24], [33], [36]. This pause violates the assumption that $\hat{s}$ increases monotonically and at a constant rate, resulting in incorrect kinematic and impedance model outputs. Therefore, during S3, we decouple $\hat{s}$ from $\theta_{\text {th }}$ and instead assume that phase continues progressing at $\dot{\hat{s}}_{S 2}$ :

$$
\hat{s}=\hat{s}_{23}+\int_{0}^{\Delta t} \dot{\hat{s}}_{S 2} d \tau \text { in } \mathrm{S} 3 .
$$

This feedforward phase progression continues until a toe-off (TO) event. Although this approach limits the user's ability to stop phase progression during S3, such cases are unlikely because stopping would inhibit power delivery from the ankle during push-off. Moreover, the under-actuated dynamics of bipedal walking dictate that once the user's gravity vector passes anterior of the stance foot, the user must continue the gait cycle until the contralateral foot lands [46]. Therefore, we expect the sacrifice in direct control of phase progression during this section of the gait cycle to be negligible.

After TO, the FSM transitions to S4, where phase is again estimated via a linear scaling of $\theta_{\text {th }}$. This mapping is defined such that $\hat{s}$ increases from $\hat{s}_{\mathrm{TO}}$ towards $s_{\mathrm{MHF}}$ as $\theta_{\mathrm{th}}$ increases:

$$
\hat{s}=\frac{\theta_{\mathrm{th}}-\theta_{\mathrm{th}}^{\mathrm{TO}}}{\theta_{\mathrm{th}}^{\mathrm{MHF}}-\theta_{\mathrm{th}}^{\mathrm{TO}}}\left(s_{\mathrm{MHF}}-\hat{s}_{\mathrm{TO}}\right)+\hat{s}_{\mathrm{TO}} \quad \text { in } \mathrm{S} 4 .
$$

The FSM transitions to 55 when $\theta_{\text {th }}$ is equivalent to the average of $\theta_{\mathrm{th}}^{\mathrm{HS}}$ and $\theta_{\mathrm{th}}^{\mathrm{MHF}}$, which typically corresponds to the end of this linear section of the thigh trajectory.

Two problems typically occurred with the previous phase variable methods when $\theta_{\mathrm{th}} \approx \theta_{\mathrm{th}}^{\mathrm{MHF}}$, which occurs during S5 in the new FSM. First, a pause in $\hat{s}$ would occur as $\dot{\theta}_{\text {th }}$ slowed and $\theta_{\mathrm{th}}$ approached $\theta_{\mathrm{th}}^{\mathrm{MHF}}$, similar to the effect seen in S3. Second, the previous methods assumed that $\theta_{\mathrm{th}}^{\mathrm{MHF}}=\theta_{\mathrm{th}}^{\mathrm{HS}}$. In cases where $\theta_{\mathrm{th}}^{\mathrm{MHF}}>\theta_{\mathrm{th}}^{\mathrm{HS}}$, such as the trajectory shown in Fig. 11 the resulting $\hat{s}$ would saturate prematurely. Excessive saturation in the phase variable can cause desynchronization between 
TABLE III

SYMBOL DEFINITIONS FOR FEATURES USED TO CALCULATE $\hat{s}$
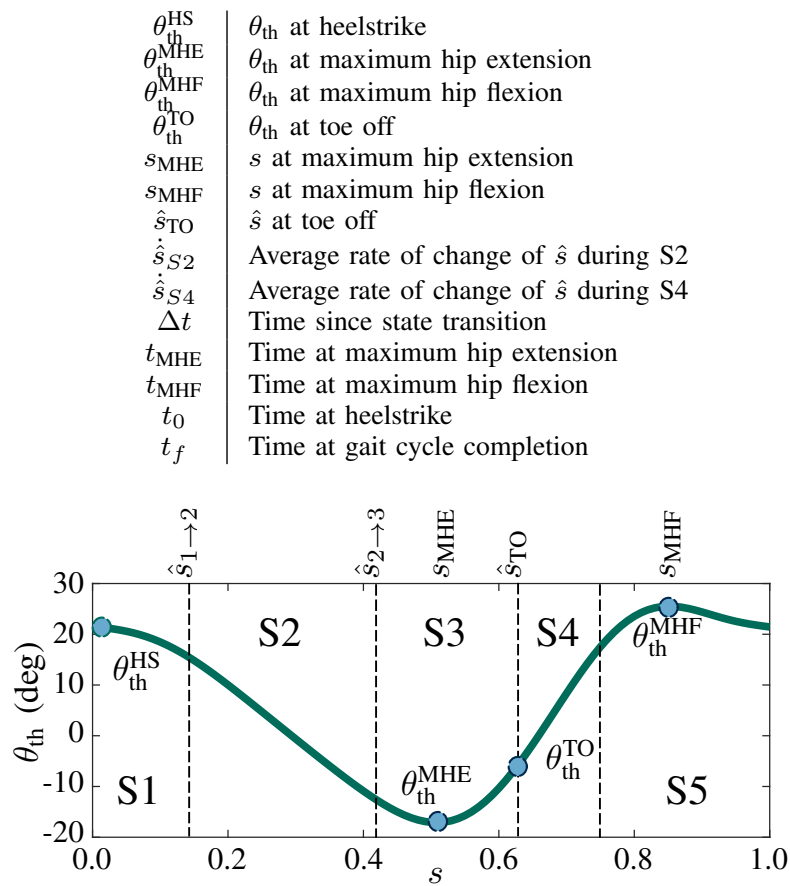

Fig. 11. The average global thigh angle trajectory $\theta_{\text {th }}$ (positive flexion) for $1 \mathrm{~m} / \mathrm{s} 0$ deg able-bodied walking, segmented by the phase variable FSM states. The phase variable is defined by linear mappings of $\theta_{\text {th }}$ during S1, $\mathrm{S} 2$ and S4, and by a feedforward phase variable rate during S3 and S5. The feedforward rates for S3 and S5 are given by the average rate of change of the phase estimates during the preceding states, which correspond to periods of constant thigh angular velocity.

the prosthesis and the user, leading to problems such as toestubbing. This effect was most exaggerated during declined walking, as the difference between $\theta_{\mathrm{th}}^{\mathrm{MHF}}$ and $\theta_{\mathrm{th}}^{\mathrm{HS}}$ was most pronounced [21]. To avoid both excessive saturation and a phase variable pause, a feedforward phase progression is again enforced based on the average phase rate in S4, $\dot{\hat{s}}_{S 4}$ :

$$
\hat{s}=\hat{s}_{45}+\int_{0}^{\Delta t} \dot{\hat{s}}_{S 4} d \tau \text { in S5. }
$$

This feedforward phase rate continues until either a heelstrike occurs or $\hat{s}=1$. If the user is walking consistently and the $\theta_{\text {th }}$ trajectory feature estimates are correct, $\hat{s}=1$ should occur simultaneously with heelstrike, returning the FSM to S1. If $\hat{s}=1$ prior to HS, the FSM transitions to S6.

S6 is primarily encountered if the user pauses at the end of the gait cycle, so it does not appear in Fig. 11. During S6, $\hat{s}$ is again calculated using a linear scaling of $\theta_{\text {th }}$, giving the user volitional control of $\hat{s}$ through $\theta_{\mathrm{th}}$ :

$$
\hat{s}=\frac{\theta_{\mathrm{th}}-\theta_{\mathrm{th}}^{\mathrm{MHE}}}{\theta_{\mathrm{th}}^{\mathrm{HS}}-\theta_{\mathrm{th}}^{\mathrm{MHE}}}\left(1-s_{\mathrm{MHE}}\right)+s_{\mathrm{MHE}} \quad \text { in S6. }
$$

This volitional control during S6 is important because it allows movements such as kicking and non-steady leg swinging [33]. As in S5, a HS event returns the FSM to S1.

2) Thigh Trajectory Feature Prediction: The $\theta_{\text {th }}$ features used in (22)-26) vary from stride-to-stride with changes in speed, incline, and natural gait variation. Some of these

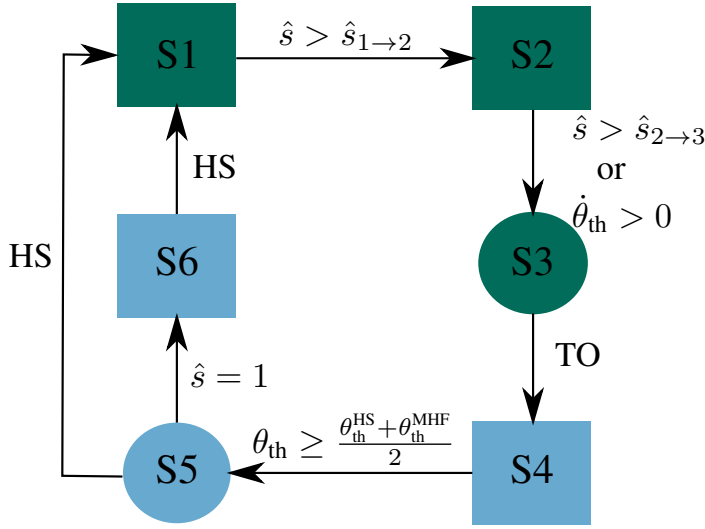

Fig. 12. Flow chart depicting the FSM states and transition criteria used in the phase variable calculation. States 1-3 (green) occur during the stance phase and states 4-6 (blue) occur during swing. States where phase is directly calculated based on thigh angle are shown as squares and states with feedforward definitions are shown as circles. State 6 is only necessary for non-steady gait and is typically bypassed during steady walking.

features are used in the phase estimate calculation before they occur in the gait cycle, specifically $\theta_{\mathrm{th}}^{\mathrm{HS}}, \theta_{\mathrm{th}}^{\mathrm{MHE}}, \theta_{\mathrm{th}}^{\mathrm{MHF}}, s_{\mathrm{MHE}}$ and $s_{\text {MHF }}$. For example, $\theta_{\mathrm{th}}^{\mathrm{MHE}}$ is used to calculate $\hat{s}$ during $\mathrm{S} 1$ and S2, but it does not typically occur until S3. Therefore, we predict these features in real-time based on observations from recent strides. At controller initialization, estimates of the thigh trajectory features are calculated using able-bodied data [21] and updated as new strides became available.

Previous work showed that care must be exercised when predicting features of the thigh trajectory to prevent unwanted interaction between the prediction algorithms and the user's gait progression. For example, [36] observed that if a simple moving average was used to calculate $\theta_{\mathrm{th}}^{\mathrm{MHE}}$, a divergent behavior occurred that resulted in the user taking progressively larger strides. To avoid this behavior, the kinematic features $\theta_{\mathrm{th}}^{\mathrm{HS}}, \theta_{\mathrm{th}}^{\mathrm{MHE}}$, and $\theta_{\mathrm{th}}^{\mathrm{MHF}}$ were estimated using a combined median and moving average filter. This filter recorded the previous 5 strides and averaged the median 3 feature values, thus rejecting non-representative strides. The history window of 5 strides balanced between filter response time and variance rejection.

Another closed-loop interaction was observed during pilot studies regarding the predictions of $s_{\mathrm{MHE}}$ and $s_{\mathrm{MHF}}$. In cases when the feature predictors were updating following a rapid change in task, we observed rare strides where $\hat{s}$ underestimated the true phase at the end of the gait cycle, causing the knee joint to not fully extend before heelstrike. Participants instinctively responded by asymmetrically extending the late swing portion of the gait cycle to try force the knee to full extension. Moving average estimates of $s_{\mathrm{MHE}}$ and $s_{\mathrm{MHF}}$, like those used for the kinematic features, caused $s_{\mathrm{MHE}}$ and $s_{\mathrm{MHF}}$ to decrease, resulting in further underestimation of $\hat{s}$ on the subsequent stride. We suspect that participants behaved this way because they were accustomed to passive prostheses, which will collapse upon loading if the knee is not fully extended. Therefore, new prediction methods were developed for $s_{\mathrm{MHE}}$ and $s_{\mathrm{MHF}}$ that favored $\hat{s}$ saturation over underestimation to combat this instinctive behavior. Let $t_{\hat{s}=1}$ be the first time during the stride that $\hat{s}=1$. Then, the $s_{\mathrm{MHE}}$ and $s_{\mathrm{MHF}}$ 
estimates were calculated as

$$
\begin{aligned}
& s_{\mathrm{MHE}}=\frac{1}{2}\left(\frac{t_{\mathrm{MHE}}-t_{0}}{t_{f}-t_{0}}+\frac{t_{\mathrm{MHE}}-t_{0}}{t_{f}-t_{\hat{s}=1}}\right), \\
& s_{\mathrm{MHF}}=\frac{1}{2}\left(\frac{t_{\mathrm{MHF}}-t_{0}}{t_{f}-t_{0}}+\frac{t_{\mathrm{MHF}}-t_{0}}{t_{f}-t_{\hat{s}=1}}\right) .
\end{aligned}
$$

The first quotient in each line of (27) is the true phase where $\theta_{\mathrm{th}}^{\mathrm{MHE}}$ and $\theta_{\mathrm{th}}^{\mathrm{MHF}}$ occurred. The second quotient is an upper bound on this true phase. We average the two so that $\hat{s}$ favors saturation and full knee extension in late swing, avoiding the potential unstable feedback loop with the user's instinctive compensations. Eventually, as $t_{\hat{s}=1} \rightarrow t_{f}$ in steady-state, the two quotients become equal and $\hat{s}$ no longer saturates. The results of (27) were likewise low-pass filtered with an infinite impulse response (IIR) filter to reject stride-to-stride variation and to prevent step changes in the estimates.

3) Phase Variable Linearization: The phase variable described above produces a consistent phase estimate trajectory over each stride during steady walking. This consistency allows a linearization map to be formed in order to further improve the phase estimate. Once the $\theta_{\text {th }}$ feature predictions converged to steady values, the average progression of $\hat{s}$ was recorded for each steady walking stride and low-pass filtered to produce an average trajectory, $\overline{\hat{s}}$. The time constant of the IIR low-pass filter was chosen to be sufficiently slow (19 strides) such that the transients of the $\theta_{\text {th }}$ feature predictors were rejected. As a further precaution, any saturated portions of $\hat{s}$ were discarded prior to averaging, as they diminish as the $\theta_{\text {th }}$ trajectory feature predictions converge.

The average phase was written as a function of true phase, given by $\overline{\hat{s}}=\sigma(s)$. Although the shape of the thigh trajectory may cause $\sigma(s)$ to be nonlinear, it is monotonic during normal walking. This implies that an inverse relationship $s=\sigma^{-1}(\overline{\hat{s}})$ exists, which can be applied to correct for nonlinearities in $\hat{s}$. First, $\sigma(s)$ was fit with a 6th order polynomial $\bar{\sigma}(s)$ that was constrained with a minimum slope of 0.2 . This minimum slope ensured strict monotonicity and numerical stability of the inverse. At each HS event, $\bar{\sigma}(s)$ was recalculated to incorporate the previous stride's effect on $\overline{\hat{s}}$. Then the final, linearized phase estimate was calculated by applying the inverse map $\bar{\sigma}^{-1}$ to the results of 22$\}-(26)$.

\section{B. Benchmark FSM Impedance Controller}

The Finite State Machine controller (FSMC), based on the FSM impedance controller presented in [15], was constructed to provide a benchmark with which to compare the HKIC. The flow of the FSMC's state machine is depicted in Figure 13 A tunable center of pressure threshold, $\ell_{\text {cop }}^{*}$, controlled the transition from S1 to S2. Then, after a tunable duration, $t_{2 \rightarrow 3}$, the FSM transitioned to S3. Next, a TO event triggered the transition to S4. Finally, knee extension $\left(\dot{\theta}_{\mathrm{k}}<0\right)$ caused a transition to S5, where the FSM remained until returning to S1 at HS. During transitions, the impedance parameters were rate-limited to prevent step changes in torque. In the FSMC, the torque command was given by 11 , where $K, B$, and $\theta_{\mathrm{eq}}$ depended on the current FSM state (given in Table IV).

Many methods have been proposed for deciding when to switch between sets of impedance parameters for different

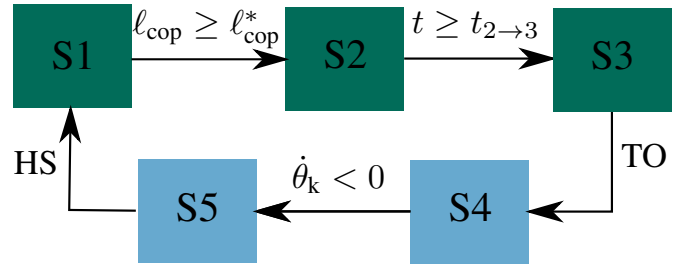

(a)

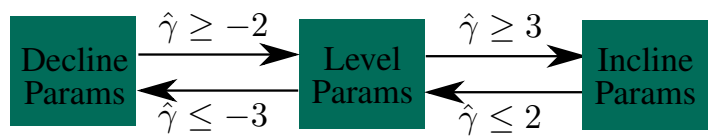

(b)

Fig. 13. (a) The structure and transition logic of the benchmark finite state machine controller. Tunable parameters $\ell_{\text {cop }}^{*}$ and $t_{2 \rightarrow 3}$ controlled the transitions from S1 to S2 and S2 to S3, while constant ground contact and knee velocity thresholds controlled the other three. States in green occur during stance and blue states during swing. (b) Task transition logic indicating how the impedance parameter sets are selected based on the incline estimate $\hat{\gamma}$.

tasks, including simple threshold methods [15] and more complex machine learning methods [47], [48]. We employed a strategy similar to [15] where the prosthesis directly estimated the ground incline using the method described in Section III-B Then a secondary FSM was used to select between parameter sets based on the estimated incline $\hat{\gamma}$. To prevent rapid switching between parameters at the boundaries, overlap was included in the switching thresholds (Fig. 13b.

TABLE IV

\begin{tabular}{|c|c|c|c|c|c|c|}
\hline \multicolumn{7}{|c|}{ SS-Level - Baseline Task $(0 \mathrm{deg}, 1 \mathrm{~m} / \mathrm{s})$} \\
\hline \multirow{2}{*}{\multicolumn{2}{|c|}{$\begin{array}{l}\text { Transition Parameters } \\
\text { Impedance Parameter }\end{array}$}} & \multicolumn{2}{|c|}{$\ell_{\mathrm{cop}}^{*}=0.08 \mathrm{~cm}$} & \multicolumn{3}{|c|}{$t_{2 \rightarrow 3}=0.091 \mathrm{~s}$} \\
\hline & & S1 & S2 & S3 & S4 & S5 \\
\hline \multirow{3}{*}{ Knee } & $K(\mathrm{Nm} / \mathrm{kg})$ & 2.25 & 2.00 & 1.00 & 1.25 & 0.75 \\
\hline & $B(\mathrm{Nms} / \mathrm{kg})$ & 0.140 & 0.126 & 0.120 & 0.070 & 0.065 \\
\hline & $\theta_{\mathrm{eq}}(\mathrm{rad})$ & 0.15 & 0.25 & 1.20 & 1.30 & -0.15 \\
\hline \multirow{3}{*}{ Ankle } & $K(\mathrm{Nm} / \mathrm{kg})$ & 1.50 & 3.50 & 4.50 & 0.10 & 0.50 \\
\hline & $B(\mathrm{Nms} / \mathrm{kg})$ & 0.140 & 0.140 & 0.140 & 0.130 & 0.100 \\
\hline & $\theta_{\mathrm{eq}}(\mathrm{rad})$ & -0.10 & -0.30 & -0.35 & 0.20 & 0.00 \\
\hline
\end{tabular}

TUNED IMPEDANCE PARAMETERS FOR EACH BASELINE TASK OF FSMC

\begin{tabular}{|c|c|c|c|c|c|c|}
\hline \multicolumn{7}{|c|}{ SS-Decline - Baseline Task $(-5 \mathrm{deg}, 1 \mathrm{~m} / \mathrm{s})$} \\
\hline \multirow{2}{*}{\multicolumn{2}{|c|}{$\begin{array}{l}\text { Transition Parameters } \\
\text { Impedance Parameters }\end{array}$}} & \multicolumn{2}{|c|}{$\ell_{\mathrm{cop}}^{*}=0.10 \mathrm{~cm}$} & \multicolumn{3}{|c|}{$t_{2 \rightarrow 3}=0.061 \mathrm{~s}$} \\
\hline & & S1 & S2 & S3 & S4 & S5 \\
\hline \multirow{3}{*}{ Knee } & $K(\mathrm{Nm} / \mathrm{kg})$ & 2.25 & 2.00 & 1.00 & 1.00 & 1.00 \\
\hline & $B(\mathrm{Nms} / \mathrm{kg})$ & 0.090 & 0.100 & 0.100 & 0.050 & 0.07 \\
\hline & $\theta_{\mathrm{eq}}(\mathrm{rad})$ & 0.15 & 0.35 & 0.75 & 1.25 & 0.05 \\
\hline \multirow{3}{*}{ Ankle } & $\bar{K}(\mathrm{Nm} / \mathrm{kg})$ & 3.50 & 3.50 & 3.50 & 1.00 & 1.00 \\
\hline & $B(\mathrm{Nms} / \mathrm{kg})$ & 0.100 & 0.100 & 0.100 & 0.100 & 0.100 \\
\hline & $\theta_{\mathrm{eq}}(\mathrm{rad})$ & -0.100 & -0.050 & 0.00 & 0.10 & 0.10 \\
\hline
\end{tabular}

\begin{tabular}{|c|l|l|l|l|l|l|}
\hline \multicolumn{7}{|c|}{ SS-Incline - Baseline Task (5 deg, 1 m/s) } \\
\hline \multicolumn{2}{|c|}{ Transition Parameters } & \multicolumn{1}{c|}{$\ell_{\mathrm{cop}}^{*}=0.05 \mathrm{~cm}$} & \multicolumn{1}{|c|}{$t_{2 \rightarrow 3}=0.190 \mathrm{~s}$} \\
\hline Impedance Parametes & $\mathrm{S} 1$ & $\mathrm{~S} 2$ & $\mathrm{~S} 3$ & $\mathrm{~S} 4$ & $\mathrm{~S} 5$ \\
\hline \multirow{3}{*}{ Knee } & $K(\mathrm{Nm} / \mathrm{kg})$ & 1.50 & 1.50 & 0.75 & 1.00 & 1.00 \\
\cline { 2 - 7 } & $B(\mathrm{Nms} / \mathrm{kg})$ & 0.090 & 0.090 & 0.090 & 0.075 & 0.065 \\
\cline { 2 - 7 } & $\theta_{\mathrm{eq}}(\mathrm{rad})$ & 0.35 & 0.25 & 0.50 & 1.20 & 0.30 \\
\hline \multirow{3}{*}{ Ankle } & $K(\mathrm{Nm} / \mathrm{kg})$ & 3.50 & 3.50 & 4.00 & 0.35 & 1.00 \\
\cline { 2 - 7 } & $B(\mathrm{Nms} / \mathrm{kg})$ & 0.140 & 0.140 & 0.140 & 0.150 & 0.100 \\
\cline { 2 - 7 } & $\theta_{\mathrm{eq}}(\mathrm{rad})$ & 0.10 & -0.10 & -0.23 & 0.20 & 0.10 \\
\hline
\end{tabular}

\section{ACKNOWLEDGEMENT}

The authors thank Vamsi Peddinti for technical assistance with the robotic leg and Leslie Wontorcik, CP, for clinical support during experiments. We also thank Dr. Kyle Embry for prior contributions to the swing kinematic model. 


\section{REFERENCES}

[1] M. R. Tucker, J. Olivier, A. Pagel, H. Bleuler, M. Bouri, O. Lambercy, J. R. Del Millán, R. Riener, H. Vallery, and R. Gassert, "Control strategies for active lower extremity prosthetics and orthotics: A review," J. Neuroeng. Rehabil., vol. 12, no. 1, 2015.

[2] J. J. Genin, G. J. Bastien, B. Franck, C. Detrembleur, and P. A. Willems, "Effect of speed on the energy cost of walking in unilateral traumatic lower limb amputees," Eur. J. of Appl. Physiol., vol. 103, 2008.

[3] D. C. Norvell, J. M. Czerniecki, G. E. Reiber, C. Maynard, J. A. Pecoraro, and N. S. Weiss, "The prevalence of knee pain and symptomatic knee osteoarthritis among veteran traumatic amputees and nonamputees," Arch. Phys. Med. Rehabil., vol. 86, no. 3, pp. 487-493, 2005.

[4] D. M. Ehde, D. G. Smith, J. M. Czerniecki, K. M. Campbell, D. M. Malchow, and L. R. Robinson, "Back pain as a secondary disability in persons with lower limb amputations," Arch. Phys. Med. Rehabil., vol. 82, no. 6, pp. 731-734, 2001.

[5] B. E. Lawson, J. Mitchell, D. Truex, A. Shultz, E. Ledoux, and M. Goldfarb, "A robotic leg prosthesis: Design, control, and implementation," IEEE Robot. Autom. Mag., vol. 21, no. 4, pp. 70-81, 2014.

[6] M. Windrich, M. Grimmer, O. Christ, S. Rinderknecht, and P. Beckerle, "Active lower limb prosthetics: A systematic review of design issues and solutions," BioMed. Eng. Online, vol. 15, 2016.

[7] B. Laschowski, J. McPhee, and J. Andrysek, "Lower-limb prostheses and exoskeletons with energy regeneration: Mechatronic design and optimization review," J. Mechanisms Robotics, vol. 11, no. 4, 052019.

[8] R. R. Torrealba and E. D. Fonseca-Rojas, "Toward the Development of Knee Prostheses: Review of Current Active Devices," Appl. Mech. Rev., vol. 71, no. 3, pp. 1-22, 2019.

[9] N. Hogan, "Impedance control: An approach to manipulation: Part i-theory," J. Dyn. Syst. Meas. Control, vol. 107, no. 1, pp. 1-7, 1985.

[10] P. M. Rack and D. R. Westbury, "The short range stiffness of active mammalian muscle and its effect on mechanical properties," J. Physiol., vol. 240, no. 2, 1974.

[11] E. Rouse, L. Hargrove, E. Perreault, and T. Kuiken, "Estimation of human ankle impedance during the stance phase of walking," IEEE Trans. Neural Syst. Rehabil. Eng., vol. 22, no. 4, pp. 870-878, 2014.

[12] H. Lee and N. Hogan, "Time-varying ankle mechanical impedance during human locomotion," IEEE Trans. Neural Syst. Rehabil. Eng., vol. 23 , no. 5, pp. 755-764, 2015.

[13] A. L. Shorter and E. J. Rouse, "Mechanical impedance of the ankle during the terminal stance phase of walking," IEEE Trans. Neural Syst. Rehabil. Eng., vol. 26, no. 1, pp. 135-143, jan 2018.

[14] F. Sup, H. Varol, J. Mitchell, T. Withrow, and M. Goldfarb, "Selfcontained powered knee and ankle prosthesis: Initial evaluation on a transfemoral amputee," IEEE Int. Conf. Rehabil. Robot., pp. 638-644, 2009.

[15] F. Sup, H. A. Varol, and M. Goldfarb, "Upslope walking with a powered knee and ankle prosthesis: Initial results with an amputee subject," IEEE Trans. Neural Syst. Rehabil. Eng., vol. 19, no. 1, pp. 71-78, 2011.

[16] A. M. Simon, K. A. Ingraham, N. P. Fey, S. B. Finucane, R. D Lipschutz, A. J. Young, and L. J. Hargrove, "Configuring a powered knee and ankle prosthesis for transfemoral amputees within five specific ambulation modes," PLoS One, vol. 9, no. 6, 2014.

[17] K. Bhakta, J. Camargo, P. Kunapuli, L. Childers, and A. Young, "Impedance control strategies for enhancing sloped and level walking capabilities for individuals with transfemoral amputation using a powered multi-joint prosthesis," Mil. Med., vol. 185, pp. 490-499, 2020.

[18] E. J. Rouse, L. M. Mooney, and H. M. Herr, "Clutchable series-elastic actuator: Implications for prosthetic knee design," Int. J. Rob. Res., vol. 33, no. 13, pp. 1611-1625, 2014.

[19] G. Hunt, S. Hood, and T. Lenzi, "Stand-Up, Squat, Lunge, and Walk with a Robotic Knee and Ankle Prosthesis under Shared Neural Control," IEEE Open J. Eng. Med. Biol., vol. 2, pp. 267-277, 2021.

[20] K. Embry, D. J. Villarreal, R. L. Macaluso, and R. D. Gregg, "Modeling the Kinematics of Human Locomotion over Continuously Varying Speeds and Inclines," IEEE Trans. Neural Syst. Rehabil. Eng., vol. 26, no. 12, pp. 2342-2350, 2018.

[21] E. Reznick, K. R. Embry, R. Neuman, E. Bolívar-Nieto, N. P. Fey, and R. D. Gregg, "Lower-limb kinematics and kinetics during continuously varying human locomotion," Sci. Data, vol. 8, 2021

[22] T. Lenzi, L. Hargrove, and J. W. Sensinger, "Preliminary evaluation of a new control approach to achieve speed adaptation in robotic transfemoral prostheses," in IEEE/RSJ Int. Conf. on Intelli. Robot. and Sys., 2014, pp. 2049-2054.
[23] T. Lenzi, M. Cempini, L. Hargrove, and T. Kuiken, "Design, development, and testing of a lightweight hybrid robotic knee prosthesis," Int. J. Rob. Res., vol. 37, no. 8, pp. 953-976, 2018.

[24] T. Elery, S. Rezazadeh, E. Reznick, L. Gray, and R. D. Gregg, "Effects of a Powered Knee-Ankle Prosthesis on Amputee Hip Compensations: A Case Series," IEEE Trans. Neural Syst. Rehabil. Eng., vol. 28, no. 12, pp. 2944-2954, 2020.

[25] H. Huang, D. L. Crouch, M. Liu, G. S. Sawicki, and D. Wang, "A Cyber Expert System for Auto-Tuning Powered Prosthesis Impedance Control Parameters," Ann. Biomed. Eng., vol. 44, no. 5, pp. 1613-1624, 2016.

[26] M. Li, Y. Wen, X. Gao, J. Si, and H. Huang, "Toward expedited impedance tuning of a robotic prosthesis for personalized gait assistance by reinforcement learning control," IEEE Trans. Robotics, vol. 38, no. 1, pp. 407-420, 2022.

[27] W. Hong, V. Paredes, K. Chao, S. Patrick, and P. Hur, "Consolidated control framework to control a powered transfemoral prosthesis over inclined terrain conditions," IEEE Int. Conf. Robot. Autom., pp. 2838$2844,2019$.

[28] A. Mohammadi and R. D. Gregg, "Variable Impedance Control of Powered Knee Prostheses Using Human-Inspired Algebraic Curves," $J$. Comput. Nonlinear Dyn., vol. 14, no. 10, pp. 1-10, 2019.

[29] N. A. Kumar, W. Hong, and P. Hur, "Impedance control of a transfemoral prosthesis using continuously varying ankle impedances and multiple equilibria," IEEE Int. Conf. Robot. Autom., pp. 1755-1761, 2020.

[30] N. Anil Kumar, S. Patrick, W. Hong, and P. Hur, "Control framework for sloped walking with a powered transfemoral prosthesis," Front. Neurorobot., vol. 15, no. Jan, pp. 1-12, 2022.

[31] F. J. Abu-Dakka and M. Saveriano, "Variable impedance control and learning-a review," Front. Robot. AI, vol. 7, 2020.

[32] N. Hogan, "Adaptive control of mechanical impedance by coactivation of antagonist muscles," IEEE Trans. on Automat. Contr., vol. 29, pp. 681-690, 1984

[33] S. Rezazadeh, D. Quintero, N. Divekar, E. Reznick, L. Gray, and R. D. Gregg, "A phase variable approach for improved rhythmic and nonrhythmic control of a powered knee-ankle prosthesis," IEEE Access, vol. 7, pp. 109 840-109855, 2019.

[34] S. Boyd and L. Vandenberghe, Convex Optimization. Cambridge University Press, 2004.

[35] D. Quintero, D. J. Villarreal, D. J. Lambert, S. Kapp, and R. D. Gregg, "Continuous-Phase Control of a Powered Knee-Ankle Prosthesis: Amputee Experiments Across Speeds and Inclines," IEEE Trans. Robot., vol. 34, no. 3, pp. 686-701, 2018

[36] T. Best, K. Embry, E. Rouse, and R. Gregg, "Phase-variable control of a powered knee-ankle prosthesis over continuously varying speeds and inclines," IEEE Int. Conf. Intelli. Robot. Sys., 2021.

[37] E. R. Westervelt, J. W. Grizzle, C. Chevallereau, J. H. Choi, and B. Morris, Feedback Control of Dynamic Bipedal Robot Locomotion. CRC Press, 2007

[38] R. Versluys, P. Beyl, M. V. Damme, A. Desomer, R. V. Ham, and D. Lefeber, "Prosthetic feet: State-of-the-art review and the importance of mimicking human ankle-foot biomechanics," Disabil. Rehabil. Assist. Tech., vol. 4, no. 2, pp. 65-75, 2009.

[39] T. Elery, S. Rezazadeh, C. Nesler, and R. D. Gregg, "Design and validation of a powered knee-ankle prosthesis with high-torque, lowimpedance actuators," IEEE Trans. Robot., vol. 36, no. 6, pp. 16491668, 2020.

[40] M. D. Fox and S. L. Delp, "Contributions of muscles and passive dynamics to swing initiation over a range of walking speeds," $J$. Biomech., vol. 43, no. 8, 2010.

[41] A. D. Kuo and J. M. Donelan, "Dynamic principles of gait and their clinical implications," Phys. Ther., vol. 90, 2010.

[42] T. Best, C. Welker, E. Rouse, and R. Gregg, "A Virtual Dog Walk with a Robotic Prosthetic Leg," 2021. [Online]. Available: https://www.youtube.com/watch?v=ozBN2ZxVxxQ

[43] M. K. Shepherd and E. J. Rouse, "Comparing Preference of Ankle-Foot Stiffness in Below-Knee Amputees and Prosthetists." Press. Nat. Sci. Reports, 2020.

[44] E. Reznick, K. Embry, and R. D. Gregg, "Predicting Individualized Joint Kinematics over a Continuous Range of Slopes and Speeds," IEEE RAS EMBS Int. Conf. Biomed. Robot. Biomech., pp. 666-672, 2020.

[45] S. M. Jaegers, J. H. Arendzen, and H. J. de Jongh, "Prosthetic gait of unilateral transfemoral amputees: A kinematic study," Arch. Phys. Med. Rehabil., vol. 76, no. 8, pp. 736-743, 1995.

[46] M. B. Popovic, A. Goswami, and H. Herr, "Ground reference points in legged locomotion: Definitions, biological trajectories and control implications," Int. J. Rob. Res., vol. 24, no. 12, pp. 1013-1032, 2005. 
[47] H. A. Varol, F. Sup, and M. Goldfarb, "Multiclass real-time intent recognition of a powered lower limb prosthesis," IEEE Trans. Biomed. Eng., vol. 57, no. 3, pp. 542-551, 2010.

[48] L. J. Hargrove, A. J. Young, A. M. Simon, N. P. Fey, R. D. Lipschutz, S. B. Finucane, E. G. Halsne, K. A. Ingraham, and T. A. Kuiken, "Intuitive control of a powered prosthetic leg during ambulation: A randomized clinical trial," J. Am. Med. Assoc., vol. 313, no. 22, pp. 2244-2252, 2015. 